\title{
A Note on the Strong Maximum Principle for Fully Nonlinear Equations on Riemannian Manifolds
}

\author{
Alessandro Goffi ${ }^{1}$ (D) . Francesco Pediconi ${ }^{2}$
}

Received: 31 July 2020 / Accepted: 8 January 2021 / Published online: 16 February 2021

(C) The Author(s) 2021

\begin{abstract}
We investigate strong maximum (and minimum) principles for fully nonlinear secondorder equations on Riemannian manifolds that are non-totally degenerate and satisfy appropriate scaling conditions. Our results apply to a large class of nonlinear operators, among which Pucci's extremal operators, some singular operators such as those modeled on the $p$ - and $\infty$-Laplacian, and mean curvature-type problems. As a byproduct, we establish new strong comparison principles for some second-order uniformly elliptic problems when the manifold has nonnegative sectional curvature.
\end{abstract}

Keywords Fully nonlinear equation · Degenerate elliptic equation · Hopf boundary lemma $\cdot$ Riemannian manifold $\cdot$ Strong maximum principle $\cdot$ Strong comparison principle

Mathematics Subject Classification Primary 35B50 $\cdot$ 35J70 $\cdot$ 58J60; Secondary 35D40 - 49L25

\footnotetext{
The first-named author is member of GNAMPA of INdAM and has been partially supported by the Fondazione CaRiPaRo Project "Nonlinear Partial Differential Equations: Asymptotic Problems and Mean-Field Games". The second-named author is member of GNSAGA of INdAM and has been supported by the project PRIN 2017 "Real and Complex Manifolds: Topology, Geometry and holomorphic dynamics" (code 2017JZ2SW5).

$\triangle$ Alessandro Goffi

alessandro.goffi@math.unipd.it

Francesco Pediconi

francesco.pediconi@unifi.it

1 Dipartimento di Matematica "Tullio Levi-Civita”, Università di Padova Via Trieste 63, 35121

Padua, Italy

2 Dipartimento di Matematica e Informatica "Ulisse Dini", Università di Firenze Viale Morgagni 67/A, 50134 Florence, Italy
} 


\section{Introduction}

This paper is devoted to analyze strong maximum and comparison principles for viscosity solutions to fully nonlinear second order equations on (finite dimensional) Riemannian manifolds $(M, g)$ of the general form

$$
F\left(x, u, \mathrm{~d} u, D^{2} u\right)=0 \quad \text { in } \Omega \subset M,
$$

being $\Omega$ a connected open subset and $F: J^{2} M \rightarrow \mathbb{R}$ proper, namely non-decreasing in the second entry and non-increasing in the last entry. Here, $J^{2} M$ denotes the 2-jet bundle over $M$ (see Sect. 3.1). More precisely, we are concerned with proving the following main results:

(SMP) Any upper semicontinuous viscosity subsolution to (1.1) attaining a nonnegative interior maximum is constant (see Theorem 4.2);

(SmP) Any lower semicontinuous viscosity supersolution to (1.1) attaining a nonpositive interior minimum is constant (see Theorem 4.7);

and one of their consequences, namely the following tangency principle:

(SCP) Let $u, v$ be respectively a viscosity sub- and supersolution to (1.1) such that $u \leq v$ in $\Omega$ and $u\left(x_{\mathrm{o}}\right)=v\left(x_{\mathrm{o}}\right)$ at some $x_{\mathrm{o}} \in \Omega$, then $u \equiv v$ in the whole $\Omega$ (see Theorem 5.4).

The first aim of this note is to complete and extend well-known results valid in the classical Euclidean case to the more general realm of Riemannian manifolds. Our second motivation is then to lay the groundwork for investigating (one-side) Liouville-type results for fully nonlinear elliptic problems as (1.1) on general Riemannian structures. This would be mainly inspired by the recent nonlinear studies in [6], which make use of (SMP) and (SmP), and the linear Liouville properties given in [32], which are intimately connected with the stochastic completeness of the manifold (cf [32, Sect. 13.2]), see also [44,54]. These latter properties will be matter of a future research by the authors and we believe this note would be a starting point to address these issues. Before stating our main results, we begin with a glimpse on the literature on maximum principles, starting with (SMP)-(SmP) and concluding with (SCP).

The (SMP) and (SmP) for linear equations

$$
L u=-\operatorname{Tr}\left(A(x) D^{2} u\right)+b(x) \cdot \nabla u+c(x) u=0 \quad \text { in } \Omega \subset \mathbb{R}^{n}
$$

with $A$ uniformly elliptic, $b$ bounded and continuous and $c$ nonnegative and bounded, date back to Hopf and it is a consequence of his Boundary Point Lemma (see e.g. $[31,50,53])$. A refinement of this procedure was implemented by E. Calabi for semicontinuous viscosity solutions to linear equations [23]. Instead, the literature on quasi-linear problems in $\mathbb{R}^{n}$, mostly modeled on $p$-Laplacian operators, is huge and we refer the interested reader to [53] for a comprehensive exposition and [52, Thm 8.1] for maximum principles under mild assumptions on the quasi-linear operator.

Let us now mention related results for fully nonlinear problems close to our model PDEs. The (SMP) and (SmP) were found by Caffarelli and Cabré [21, Prop 4.9] as 
a consequence of the weak Harnack inequality. Under lower ellipticity conditions on the nonlinear operator $F$, they were derived in [7,39], the former covering even many examples of quasi-linear equations. Descriptions via control theoretic and probabilistic arguments of the propagation sets of maxima (and minima) for Hamilton-JacobiBellman equations were provided in [8,9]. Later results for fully nonlinear uniformly elliptic equations with linear gradient growth can be found in [36, Thm 5.1], see also [22] for other maximum principles for nonlinear elliptic operators. Property (SMP) has been also obtained in [2, Thm 5.1] via the weak Harnack inequality for some fully nonlinear PDEs (not necessarily uniformly elliptic) with superlinear and at most quadratic growth in the first order term.

Finally, recent contributions for fully nonlinear PDEs over Hörmander vector fields have been given in [10]. More recent progresses have been made on Hessian operators (mostly for truncated Laplacians), and they can be found in $[16,17,28]$, while a rather different approach involving fully nonlinear uniformly elliptic PDEs and quasi-linear equations perturbed by suitable lower order terms has been proposed in [27,44]. Additional results on maximum principles for fully nonlinear equations can be found in the recent works [25,58], see also the references therein.

The literature of the corresponding equations on Riemannian manifolds is poor, although (SMP) and (SmP) are of local nature and one does not usually expect their failure depending on the properties of the considered manifold. For linear elliptic problems, the first result appeared in [23], see also [13, Prop 6.84] and [48, Appx A] for weak solutions to the manifold Laplace equation. The case of nonlinear equations modeled on the $p$-Laplacian perturbed by zeroth-order terms has been addressed in [53, Sect. 8.5]. A more general treatment of quasi-linear equations, involving even mean-curvature operators, can be found in [14, Sect. 4.2] under the name of finite maximum principle and in [1, Thm 3.10] for PDEs perturbed by first-order terms, see also [49] and the references therein. In the context of fully nonlinear operators, the only available results we are aware of in the context of Riemannian manifolds are those established by Harvey and Lawson [35], that however, cover the case of Hessian-type equations only.

Our approach is inspired by the landmark papers [7-9] and borrows several (viscosity) techniques from these works. In particular, we stress that the procedure implemented here is structured in the spirit of that used by Calabi [23] and the nonlinear one by Bardi and Da Lio [7], where solutions are meant in the weak viscosity sense, unlike the distributional framework developed in [3,53]. Our main result (SMP) that we prove in Theorem 4.2 for (1.1) is based on a classical barrier-type argument for viscosity solutions and works for those operators $F$ that are elliptic according to the following notion

$$
\begin{aligned}
& \sup _{\alpha>0} F(x, 0, q, Q-\alpha q \otimes q)>0 \quad \text { for any } x \in \Omega, \\
& q \in T_{x}^{*} M, q \neq 0, \quad Q \in \operatorname{Sym}^{2}\left(T_{x} M\right)
\end{aligned}
$$

and fulfilling a scaling condition like

$$
F(x, c s, c q, c Q) \geq \eta(c) F(x, s, q, Q) \quad \text { for any } s \in[-1,0] \text { and } c \in(0,1],
$$


for some function $\eta>0$ (see Sect. 3.1 for detailed assumptions). In particular, we highlight that the ellipticity condition reduces to the non-degeneracy property identified by Bardi and Da Lio [7] in the Euclidean setting whenever the manifold has nonnegative sectional curvature (see Remark 4.3). In Sects. 3.2 and 4 we list several examples to which our main Theorem 4.2 applies, among which equations driven by Pucci's, $p$-Laplace Beltrami, $\infty$-Laplacian and mean curvature operators, together with various singular nonlinear operators modeled on the $p$ - and $\infty$-Laplacian for which the viscosity theory on Riemannian manifolds has been developed, see e.g. [41]. We will also provide a weak version of the Hopf boundary Lemma for viscosity solutions in Theorem 4.5. Furthermore, symmetric ellipticity and scaling conditions lead to a version of the (SmP), that is stated in Theorem 4.7 for reader's convenience.

Let us now pass to discuss property (SCP), for which the literature is less wide. For classical solutions to linear equations it is a straightforward byproduct of the (SMP). In the case of nonlinear problems, even degenerate, some additional conditions on the operator are needed. Property (SCP) for smooth solutions to quasi-linear equations can be found in [53, Thm 2.2.2] under the name of tangency principle. In the fully nonlinear case, strong comparison principles have been addressed in [7, Rem 3] (when one of the functions is $\mathcal{C}^{2}$ via the arguments in [39]), by Ishii-Yoshimura in [36, Thm 5.3] for second order uniformly elliptic equations with Lipschitz growth in $(u, \mathrm{~d} u)$, N.S. Trudinger [56] for Lipschitz continuous viscosity solutions, Y. Giga and M. Onhuma [30] for semicontinuous solutions, see also [46, Thm 3.1] for other results on uniformly elliptic operators and [45] for the case of mean curvature equations, all of them in the Euclidean setting. More recently, strong comparison principles have been proved in [10] for degenerate Hamilton-Jacobi-Bellman equations, covering also problems structured over Hörmander vector fields, see also [42] for tangency principles results for fully nonlinear problems arising in conformal geometry.

In Sect. 5, we first set up a simple proof for Pucci's extremal operators when one of the involved functions is smooth (see Lemma 5.1) and then we prove (SCP) following a strategy implemented in [36] based on a combination of the (SMP) with the weak comparison principle in [4] that yields the result in the uniformly elliptic case. In particular, we assume $F$ intrinsically uniformly continuous, uniformly elliptic in the sense that

$$
\lambda \operatorname{Tr}(P) \leq F(x, s, q, Q)-F(x, s, q, Q+P) \leq \Lambda \operatorname{Tr}(P)
$$

for any $x \in M,(s, q, Q) \in \widetilde{J}_{x}^{2} M, P \in \operatorname{Sym}^{2}\left(T_{x} M\right)$ with $P \geq 0$ and ellipticity constants $0<\lambda \leq \Lambda$, and satisfying

$$
\left|F\left(x, s_{1}, q_{1}, Q\right)-F\left(x, s_{2}, q_{2}, Q\right)\right| \leq C\left(\left|s_{1}-s_{2}\right|+\left|q_{1}-q_{2}\right|\right)
$$

for any $x \in M$ and $\left(s_{1}, q_{1}, Q\right),\left(s_{2}, q_{2}, Q\right) \in J_{x}^{2} M$, and prove (SCP) whenever the manifold has nonnegative sectional curvature, see Remark 5.7 for further comments on this restriction. This latter result is contained in Theorem 5.4 and essentially relies on proving that the difference $w=u-v$ is a subsolution to a sort of linearized equation, following the path of [36]. 
As far as the weak comparison principle is concerned, the literature is huge when $F$ is strictly proper since the results encompass even first order problems $[5,26]$. In the more general case of Riemannian manifolds, the only available contributions we are aware of in the second-order case have been obtained in [4]. In particular, when the manifold has nonnegative sectional curvature, weak comparison principles are obtained via the Riemannian counterpart of the Euclidean theorem on sums [26], see [4]. However, when $(M, g)$ has negative curvature, the weak comparison continues to hold under a further uniform continuity assumption (see [4, cond (2b) on Cor 4.10] and Remark 5.7 below). We refer also to [43, Appx A] for further comparison principles on Riemannian manifolds for quasi-linear problems.

Under the mere properness of the operator one needs some form of ellipticity and the minimal conditions seem to be an open problem, see $[12,37,39,40]$ and the more recent $[10,11,25]$.

We finally mention that other kind of maximum principles (at infinity) on Riemannian manifolds can be found in the monographs [33,49], while AlexandroffBakelman-Pucci estimates together with maximum principles for subsolutions of linear problems were obtained in [20].

Plan of the paper Section 2 is devoted to some preliminaries on Differential Geometry. Section 3 introduces fully nonlinear second order equations on Riemannian manifolds together with the ellipticity definitions used throughout the paper. Section 4 comprehends the proof of the (SMP) and (SmP) with a list of prototype examples, while Sect. 5 applies the results to deduce (SCP).

Acknowledgement We thank Prof. Hitoshi Ishii for providing us with a copy of [36]. We also thank the anonymous referees for their careful reading of the manuscript and useful comments.

\section{Preliminaries of Riemannian Geometry}

Let $(M, g)$ be a $\mathcal{C}^{3}$-Riemannian $n$-manifold, i.e. a connected smooth manifold $M$ of dimension $n$ endowed with a symmetric $(0,2)$-tensor field $g \in \mathcal{C}^{3}\left(M, \operatorname{Sym}^{2}\left(T^{*} M\right)\right)$, which induces at each point $x \in M$ a positive definite inner product $g_{x}$ on the tangent space $T_{x} M$. We denote by d the exterior derivative on $M$ and by $D$ the Levi-Civita covariant derivative on $(M, g)$. We call domain any connected open subset $\Omega \subset M$. If the closure $\bar{\Omega} \subset M$ is compact, we say that $\Omega$ has compact closure.

From now on, all the manifolds are assumed to be connected, all the Riemannian metrics are assumed to be of class $\mathcal{C}^{3}$ and they are not necessarily complete.

Let $\Omega \subset M$ be a domain. Given a function $\varphi \in \mathcal{C}^{2}(\Omega, \mathbb{R})$, we denote:

- by $\nabla \varphi \in \mathcal{C}^{1}\left(\Omega,\left.T M\right|_{\Omega}\right)$ the gradient of $\varphi$, which is defined by

$$
g(\nabla \varphi, X):=\mathrm{d} \varphi(X) \text { for any } X \in \mathcal{C}^{\infty}\left(\Omega,\left.T M\right|_{\Omega}\right)
$$

- by $D^{2} \varphi \in \mathcal{C}^{0}\left(\Omega,\left.\operatorname{Sym}^{2}\left(T^{*} M\right)\right|_{\Omega}\right)$ the Hessian of $\varphi$, which is defined by $D^{2} \varphi:=$ $D(\mathrm{~d} \varphi)$, i.e. 


$$
D^{2} \varphi(X, Y)=X(\mathrm{~d} \varphi(Y))-\mathrm{d} \varphi\left(D_{X} Y\right) \quad \text { for any } X, Y \in \mathcal{C}^{\infty}\left(\Omega,\left.T M\right|_{\Omega}\right) .
$$

The Riemannian manifold $(M, g)$ is in particular a separable, locally compact length space by means of the induced Riemannian distance dist : $M \times M \rightarrow \mathbb{R}$ (see e.g. [47, Sect. 2]). We will denote by $B(x, r)$ the open metric ball centered at $x \in M$ of radius $r>0$ in $M$, by $S(x, r):=\partial B(x, r)$ its boundary and by $\bar{B}(x, r):=\overline{B(x, r)}$ its closure. Notice that, by the classical Hopf-Rinow Theorem, if $(M, g)$ is complete, then a domain has compact closure if and only if it is bounded (see e.g. [19, Thm 2.5.28]). Moreover, for any $x \in M$, we denote by $\operatorname{Exp}_{x}$ the Riemannian exponential map at $x$ and by $\operatorname{inj}(x)$ the injectivity radius of $(M, g)$ at $x$.

Let $x_{\mathrm{o}} \in M$ be a point. We denote by $|\cdot|$ the norm induced by $g$ on the tangent bundle $T M$ and by $B\left(0_{x_{0}}, \operatorname{inj}\left(x_{\mathrm{o}}\right)\right) \subset T_{x_{0}} M$ the ball centered at the origin of the tangent space $T_{x_{0}} M$ with radius $\operatorname{inj}\left(x_{\mathrm{o}}\right)$. Then, it is known that the restricted map

$$
\operatorname{Exp}_{x_{\mathrm{o}}}: B\left(0_{x_{\mathrm{o}}}, \operatorname{inj}\left(x_{\mathrm{o}}\right)\right) \subset T_{x_{\mathrm{o}}} M \rightarrow B\left(x_{\mathrm{o}}, \operatorname{inj}\left(x_{\mathrm{o}}\right)\right) \subset M
$$

is a diffeomorphism of class $\mathcal{C}^{2}$ and the function

$$
f_{x_{0}}: B\left(x_{\mathrm{o}}, \operatorname{inj}\left(x_{\mathrm{o}}\right)\right) \rightarrow \mathbb{R}, \quad f_{x_{\mathrm{o}}}(x):=\frac{1}{2} \operatorname{dist}\left(x, x_{\mathrm{o}}\right)^{2}
$$

is of class $\mathcal{C}^{2}$ with $\left(\mathrm{d} f_{x_{0}}\right)(x) \neq 0_{x}$ for any $x \in B\left(x_{\mathrm{o}}, \operatorname{inj}\left(x_{\mathrm{o}}\right)\right), x \neq x_{\mathrm{o}}$. This follows from the Gauss Lemma, which implies that $\operatorname{dist}\left(x, x_{0}\right)=\left|\left(\operatorname{Exp}_{x_{0}}\right)^{-1}(x)\right|$ for any $x \in B\left(x_{\mathrm{o}}, \operatorname{inj}\left(x_{\mathrm{o}}\right)\right)$.

We recall that the injectivity radius $x \mapsto \operatorname{inj}(x)$ is continuous whenever $g$ is complete (see e.g. [55]). Moreover, for any $0<r<\operatorname{inj}\left(x_{\mathrm{o}}\right)$, the ball $B\left(x_{\mathrm{o}}, r\right)$ has compact closure, the sphere $S\left(x_{0}, r\right)=\left(f_{x_{0}}\right)^{-1}\left(\frac{r^{2}}{2}\right)$ is an embedded $(n-1)$-submanifold of class $\mathcal{C}^{2}$ and, for any $x \in S\left(x_{0}, r\right)$, the gradient $\left(\nabla f_{x_{0}}\right)(x)$ is outward-pointing perpendicular to the tangent space $T_{x} S\left(x_{0}, r\right)$. For the sake of notation, given $x, y \in M$ such that dist $(x, y)<\min \{\operatorname{inj}(x), \operatorname{inj}(y)\}$, we denote by $L_{x y}: T_{x} M \rightarrow T_{y} M$ the parallel transport along the unique minimizing geodesic from $x$ to $y$.

Let now $x_{\mathrm{o}} \in M$ and $0<r<\operatorname{inj}\left(x_{\mathrm{o}}\right)$. We recall that the ball $B\left(x_{\mathrm{o}}, r\right)$ is said to be strongly geodesically convex if, for any ball $B\left(y, r^{\prime}\right) \subset B\left(x_{0}, r\right)$, any two points in $B\left(y, r^{\prime}\right)$ are joined by a unique minimizing geodesic which is entirely contained in $B\left(y, r^{\prime}\right)$. In this case, it holds that $\left(D^{2} f_{x_{0}}\right)(x)>0$ for any $x \in B\left(x_{0}, r\right)$. The value

$$
R\left(x_{\mathrm{o}}\right):=\sup \left\{0<r<\operatorname{inj}\left(x_{\mathrm{o}}\right): B\left(x_{\mathrm{o}}, r\right) \text { is strongly geodesically convex }\right\}
$$

is called convexity radius of $(M, g)$ at $x_{0}$. We recall that the function $x \mapsto R(x)$ is positive and, from the very definition, it is also 1-Lipschitz (see e.g. [59]). More precisely:

- if there exists $\tilde{x} \in M$ such that $R(\tilde{x})=+\infty$, then $R(x)=+\infty$ for any $x \in M$;

- if there exists $\tilde{x} \in M$ such that $R(\tilde{x})<+\infty$, then $R(x)<+\infty$ for any $x \in M$ and

$$
|R(x)-R(y)| \leq \operatorname{dist}(x, y) \text { for any } x, y \in M \text {. }
$$


Notice that in the Euclidean case, i.e. when $(M, g)=\left(\mathbb{R}^{n},\langle\rangle,\right)$, for any $x_{\mathrm{o}} \in \mathbb{R}^{n}$ it holds that

$$
R\left(x_{\mathrm{o}}\right)=\operatorname{inj}\left(x_{\mathrm{o}}\right)=+\infty, \quad f_{x_{\mathrm{o}}}(x)=\frac{1}{2}\left|x-x_{\mathrm{o}}\right|^{2}, \quad\left(\mathrm{~d} f_{x_{\mathrm{o}}}\right)(x)=\left\langle x-x_{\mathrm{o}}, \cdot\right\rangle, \quad\left(D^{2} f_{x_{\mathrm{o}}}\right)(x)=\langle,\rangle
$$

and any parallel transport $L_{x y}: \mathbb{R}^{n} \simeq T_{x} \mathbb{R}^{n} \rightarrow T_{y} \mathbb{R}^{n} \simeq \mathbb{R}^{n}$ coincides with the identity map.

\section{Fully Nonlinear PDEs on Riemannian Manifolds}

\subsection{Notation}

Let $(M, g)$ be a Riemannian manifold and $\Omega \subset M$ a domain. We denote by $J^{2} M \rightarrow M$ the 2-jet bundle on $M$, which splits as

$$
J^{2} M=(M \times \mathbb{R}) \oplus_{M} T^{*} M \oplus_{M} \operatorname{Sym}^{2}\left(T^{*} M\right)
$$

by means of the Riemannian metric $g$. For any function $\varphi \in \mathcal{C}^{2}(\Omega, \mathbb{R})$, its 2 -jet at $x$ is given by

$$
\left(j^{2} \varphi\right)(x):=\left(\varphi(x), \mathrm{d} \varphi(x), D^{2} \varphi(x)\right) \in J_{x}^{2} M, \quad J_{x}^{2} M=\mathbb{R} \oplus T_{x}^{*} M \oplus \operatorname{Sym}^{2}\left(T_{x}^{*} M\right) .
$$

We define the subbundle $\widetilde{J}^{2} M \subset J^{2} M$ by setting

$$
\widetilde{J}^{2} M:=\bigsqcup_{x \in M} \widetilde{J}_{x}^{2} M, \quad \widetilde{J}_{x}^{2} M:=\left\{(s, q, Q) \in J_{x}^{2}(M): q \neq 0\right\} .
$$

Let us consider a function $F: \widetilde{J}^{2}(M) \rightarrow \mathbb{R}$, which is assumed to satisfy the following fundamental condition:

(p.) $F$ is proper, i.e. $F(x, r, q, P) \leq F(x, s, q, Q)$ whenever $r \leq s$ and $Q \leq P$.

For the sake of shortness, for any function $\varphi \in \mathcal{C}^{2}(\Omega, \mathbb{R})$ we define

$$
\begin{aligned}
F[\varphi](x) & :=F\left(x,\left(j^{2} \varphi\right)(x)\right) \\
& =F\left(x, \varphi(x), \mathrm{d} \varphi(x), D^{2} \varphi(x)\right) \text { for any } x \in \Omega \text { such that } D \varphi(x) \neq 0 .
\end{aligned}
$$

Moreover, we set

$$
\begin{aligned}
& \operatorname{USC}(\Omega):=\{\text { upper semicontinuous functions } u: \Omega \rightarrow[-\infty,+\infty)\} \\
& \operatorname{LSC}(\Omega):=\{\text { lower semicontinuous functions } v: \Omega \rightarrow(-\infty,+\infty]\}
\end{aligned}
$$

and we define $\operatorname{USC}(\bar{\Omega}), \operatorname{LSC}(\bar{\Omega})$ similarly. Then, we recall the definitions of viscosity sub- and supersolution of the equation

$$
F[u](x)=F\left(x, u(x), \mathrm{d} u(x), D^{2} u(x)\right)=0 \quad \text { on } \Omega .
$$


Definition 3.1 A function $u \in \operatorname{USC}(\Omega)$ (resp. $v \in \operatorname{LSC}(\Omega)$ ) is a viscosity subsolution (resp. viscosity supersolution) to (3.1) if, for any function $\varphi \in \mathcal{C}^{2}(\Omega, \mathbb{R})$ and $x \in$ $\Omega$ local maximum of $u-\varphi$ (resp. local minimum of $v-\varphi$ ) with $\mathrm{d} \varphi(x) \neq 0$, it holds $F\left(x, u(x), \mathrm{d} \varphi(x), D^{2} \varphi(x)\right) \leq 0$ (resp. $F\left(x, v(x), \mathrm{d} \varphi(x), D^{2} \varphi(x)\right) \geq 0$ ). A continuous function $u: \Omega \rightarrow \mathbb{R}$ is a viscosity solution to (3.1) if it is both a viscosity subsolution and a viscosity supersolution.

Remark 3.2 Notice that, since we allow $F$ to be singular at $q=0$, this definition is slightly weaker than the one in [26, Sect. 2]. Other refined notions of viscosity inequalities via the envelopes have appeared when dealing with singular equations (we refer e.g. to [29, Def 2.3.1], [41] and references therein). However, we stress that if $F$ is continuous and $u: \Omega \rightarrow \mathbb{R}$ is of class $\mathcal{C}^{2}$ with $\mathrm{d} u \neq 0$, then $u$ is a viscosity subsolution (resp. viscosity supersolution) to $F[u]=0$ if and only if it is a classical subsolution (resp. classical supersolution) to $F[u]=0$.

We introduce now two notions of ellipticity which will be of central importance in this work (see also [7, Sec 1]). These are:

(1.p.e.) $F$ is lower partially elliptic if for any $x_{\mathrm{o}} \in M$ there exists a function $\alpha_{x_{\mathrm{o}}}$ : $B\left(x_{\mathrm{o}}, R\left(x_{\mathrm{o}}\right)\right) \rightarrow[0,+\infty)$ such that

$$
F\left(x, 0,\left(\mathrm{~d} f_{x_{0}}\right)(x),\left(D^{2} f_{x_{0}}\right)(x)-\alpha\left(\mathrm{d} f_{x_{\mathrm{o}}}\right)(x) \otimes\left(\mathrm{d} f_{x_{\mathrm{o}}}\right)(x)\right)>0
$$

for any $x \in B\left(x_{\mathrm{o}}, R\left(x_{\mathrm{o}}\right)\right), x \neq x_{\mathrm{o}}$, and $\alpha>\alpha_{x_{\mathrm{o}}}(x)$;

(u.p.e.) $F$ is upper partially elliptic if for any $x_{\mathrm{o}} \in M$ there exists a function $\alpha_{x_{\mathrm{o}}}$ : $B\left(x_{0}, R\left(x_{0}\right)\right) \rightarrow[0,+\infty)$ such that

$$
F\left(x, 0,\left(\mathrm{~d} f_{x_{\mathrm{o}}}\right)(x), \alpha\left(\mathrm{d} f_{x_{\mathrm{o}}}\right)(x) \otimes\left(\mathrm{d} f_{x_{\mathrm{o}}}\right)(x)-\left(D^{2} f_{x_{\mathrm{o}}}\right)(x)\right)<0
$$

for any $x \in B\left(x_{\mathrm{o}}, R\left(x_{\mathrm{o}}\right)\right), x \neq x_{\mathrm{o}}$, and $\alpha>\alpha_{x_{\mathrm{o}}}(x)$;

Here, notice that $R\left(x_{\mathrm{o}}\right)$ and $f_{x_{0}}$ have been defined in (2.2) and (2.1), respectively. For the sake of shortness, we say that $F$ is partially elliptic if it is both lower and upper partially elliptic. Furthermore, we recall that $F$ is uniformly elliptic if there exist two constants $0<\lambda \leq \Lambda$ such that

$$
\lambda \operatorname{Tr}(P) \leq F(x, s, q, Q)-F(x, s, q, Q+P) \leq \Lambda \operatorname{Tr}(P)
$$

for any $x \in M,(s, q, Q) \in \widetilde{J}_{x}^{2} M, P \in \operatorname{Sym}^{2}\left(T_{x} M\right)$ with $P \geq 0$. Notice that uniform ellipticity implies partial ellipticity (see Lemma 3.4), but the converse assertions do not hold true.

We also introduce two scaling properties which will play a role (see again [7, Sec 1]). These are:

(1.s.p.) $F$ has the lower scaling property if for any $x_{\mathrm{o}} \in M$ there exist two functions $\eta_{x_{\mathrm{o}}}:(0,1] \rightarrow(0,+\infty)$ and $\alpha_{x_{\mathrm{o}}}: B\left(x_{\mathrm{o}}, R\left(x_{\mathrm{o}}\right)\right) \rightarrow[0,+\infty)$ such that

$$
\begin{aligned}
& F\left(x, c s, c\left(\mathrm{~d} f_{x_{0}}\right)(x), c\left(\left(D^{2} f_{x_{0}}\right)(x)-\alpha\left(\mathrm{d} f_{x_{0}}\right)(x) \otimes\left(\mathrm{d} f_{x_{0}}\right)(x)\right)\right) \geq \\
& \eta_{x_{0}}(c) F\left(x, s,\left(\mathrm{~d} f_{x_{0}}\right)(x),\left(D^{2} f_{x_{0}}\right)(x)-\alpha\left(\mathrm{d} f_{x_{0}}\right)(x) \otimes\left(\mathrm{d} f_{x_{0}}\right)(x)\right)
\end{aligned}
$$


for any $c \in(0,1], s \in[-1,0], x \in B\left(x_{0}, R\left(x_{0}\right)\right), x \neq x_{\mathrm{o}}$ and $\alpha>\alpha_{x_{0}}(x)$;

(u.s.p.) $F$ has the upper scaling property if for any $x_{\mathrm{o}} \in M$ there exist two functions

$\eta_{x_{\mathrm{o}}}:(0,1] \rightarrow(0,+\infty)$ and $\alpha_{x_{\mathrm{o}}}: B\left(x_{\mathrm{o}}, R\left(x_{\mathrm{o}}\right)\right) \rightarrow[0,+\infty)$ such that

$$
\begin{aligned}
& F\left(x, c s, c\left(\mathrm{~d} f_{x_{0}}\right)(x), c\left(\alpha\left(\mathrm{d} f_{x_{0}}\right)(x) \otimes\left(\mathrm{d} f_{x_{0}}\right)(x)-\left(D^{2} f_{x_{0}}\right)(x)\right)\right) \leq \\
& \eta_{x_{0}}(c) F\left(x, s,\left(D f_{x_{0}}\right)(x), \alpha\left(D f_{x_{0}}\right)(x) \otimes\left(D f_{x_{0}}\right)(x)-\left(D^{2} f_{x_{0}}\right)(x)\right)
\end{aligned}
$$

for any $c \in(0,1], s \in[0,1], x \in B\left(x_{\mathrm{o}}, R\left(x_{\mathrm{o}}\right)\right), x \neq x_{\mathrm{o}}$ and $\alpha>\alpha_{x_{\mathrm{o}}}(x)$.

Finally, we recall that $F$ is said to be positively $h$-homogeneous, with $h \in \mathbb{R}$, if

$$
F(x, c s, c q, c Q)=c^{h} F(x, s, q, Q)
$$

for any $c>0, x \in M,(s, q, Q) \in \widetilde{J}_{x}^{2} M$. Clearly, positive $h$-homogeneity implies both the lower and the upper scaling properties, but the converse assertions do not hold true.

\subsection{Universal Riemannian Operators}

We recall here the construction of a distinguished kind of PDEs on Riemannian manifolds. More specifically, these have constant coefficients and are obtained from some Euclidean operators via the action of the orthogonal group $\mathrm{O}(n)$ (see [34, Sect. 5]).

Let $(M, g)$ be a Riemannian manifold. Consider a function

$$
\underline{\mathcal{F}}: \mathbb{R} \times\left(\mathbb{R}^{n} \backslash\{0\}\right) \times \operatorname{Sym}(n) \rightarrow \mathbb{R}
$$

and assume that it is invariant under the standard left action of the orthogonal group $\mathrm{O}(n)$, i.e.

$$
\underline{\mathcal{F}}(s, v, A)=\underline{\mathcal{F}}\left(s, a \cdot v, a \cdot A \cdot a^{T}\right) \quad \text { for any } a \in \mathrm{O}(n) .
$$

Then, one can construct an associated operator $\mathcal{F}: \widetilde{J}^{2} M \rightarrow \mathbb{R}$ in the following way. First, for any $x \in M$ and for any orthonormal frame $e=\left(e_{1}, \ldots, e_{n}\right)$ for $T_{x} M$, we consider the linear isomorphism

$$
\Phi_{(x, e)}: J_{x}^{2} M \rightarrow \mathbb{R} \oplus \mathbb{R}^{n} \oplus \operatorname{Sym}(n), \quad \Phi_{(x, e)}(s, q, Q):=\left(s, \Phi_{(x, e)}^{1}(q), \Phi_{(x, e)}^{2}(Q)\right)
$$

defined by

$$
\Phi_{(x, e)}^{1}(q):=\left(q\left(e_{1}\right), \ldots, q\left(e_{n}\right)\right)^{T}, \quad \Phi_{(x, e)}^{2}(Q):=\left(Q\left(e_{j}, e_{\ell}\right) \delta^{\ell i}\right)_{\substack{1 \leq i \leq n \\ 1 \leq j \leq n}}
$$

Then, we define

$$
\mathcal{F}: \widetilde{J}^{2} M \rightarrow \mathbb{R}, \quad \mathcal{F}(x, s, q, Q):=\underline{\mathcal{F}}\left(\left(\Phi_{(x, e)}\right)(s, q, Q)\right),
$$


where $e=\left(e_{1}, \ldots, e_{n}\right)$ is any orthonormal frame for the tangent space $T_{x} M$. Notice that, by means of the $\mathrm{O}(n)$-invariance of $\underline{\mathcal{F}}$, one can easily prove that $\mathcal{F}$ is well defined. The function $\mathcal{F}$ is called universal Riemannian operator associated to $\mathcal{F}$. It is straightforward to check that the following statements hold true:

(i) $\mathcal{F}$ is proper/continuous/uniformly elliptic/positively $h$-homogeneous if and only if $\mathcal{F}$ is too;

(ii) $\mathcal{F}$ is lower partially elliptic if

$$
\sup _{\alpha>0} \underline{\mathcal{F}}\left(0, v, A-\alpha v \otimes v^{T}\right)>0 \quad \text { for any } v \in \mathbb{R}^{n} \backslash\{0\}, A \in \operatorname{Sym}(n)
$$

(ii') $\mathcal{F}$ is upper partially elliptic if

$$
\inf _{\alpha>0} \underline{\mathcal{F}}\left(0, v, \alpha v \otimes v^{T}-A\right)<0 \quad \text { for any } v \in \mathbb{R}^{n} \backslash\{0\}, A \in \operatorname{Sym}(n)
$$

(iii) $\mathcal{F}$ has the lower scaling property if there exists a function $\eta:(0,1] \rightarrow(0,+\infty)$ such that

$$
\begin{aligned}
& \underline{\mathcal{F}}(c s, c v, c A) \geq \eta(c) \underline{\mathcal{F}}(s, v, A) \\
& \quad \text { for any } c \in(0,1], s \in[-1,0], v \in \mathbb{R}^{n} \backslash\{0\}, A \in \operatorname{Sym}(n)
\end{aligned}
$$

(iii') $\mathcal{F}$ has the upper scaling property if there exists a function $\eta:(0,1] \rightarrow(0,+\infty)$ such that

$$
\begin{aligned}
& \underline{\mathcal{F}}(c s, c v, c A) \leq \eta(c) \underline{\mathcal{F}}(s, v, A) \\
& \quad \text { for any } c \in(0,1], s \in[0,1], v \in \mathbb{R}^{n} \backslash\{0\}, A \in \operatorname{Sym}(n)
\end{aligned}
$$

The most famous example of universal Riemannian operator is the LaplaceBeltrami operator, which is associated to the Euclidean operator

$$
\underline{\mathcal{F}}_{\mathrm{LB}}: \operatorname{Sym}(n) \rightarrow \mathbb{R}, \quad \underline{\mathcal{F}}_{\mathrm{LB}}(A):=-\operatorname{Tr}(A) .
$$

It is proper, positively 1-homogeneous, Lipschitz continuous and uniformly elliptic with ellipticity constants $\lambda=\Lambda=1$.

Remark 3.3 Another well-known example is the Monge-Ampère operator, which is associated to

$$
\underline{\mathcal{F}}_{\mathrm{MA}}: \operatorname{Sym}(n) \rightarrow \mathbb{R}, \quad \underline{\mathcal{F}}_{\mathrm{MA}}(A):=\operatorname{det}(A) .
$$

However, being the determinant not partially elliptic, it does not fall into the treatment of our analysis.

We are going to list below some other examples of universal Riemannian operators. From now on, for any $A \in \operatorname{Sym}(n)$, we will denote by $\mu_{1}(A) \leq \cdots \leq \mu_{n}(A)$ its ordered eigenvalues. 


\section{Pucci's Extremal Operators}

Fix two constants $0<\lambda \leq \Lambda$ and consider the set $\mathcal{B}_{\lambda, \Lambda}:=\{B \in \operatorname{Sym}(n): \lambda I \leq$ $B \leq \Lambda I\}$. Then, we define $\underline{\mathcal{M}}_{\lambda, \Lambda}^{ \pm}: \operatorname{Sym}(n) \rightarrow \mathbb{R}$ as

$$
\begin{aligned}
& \underline{\mathcal{M}}_{\lambda, \Lambda}^{+}(A):=\sup _{B \in \mathcal{B}_{\lambda, \Lambda}}(-\operatorname{Tr}(B . A))=-\lambda \sum_{\mu_{i}(A)>0} \mu_{i}(A)-\Lambda \sum_{\mu_{i}(A)<0} \mu_{i}(A), \\
& \underline{\mathcal{M}}_{\lambda, \Lambda}^{-}(A):=\inf _{B \in \mathcal{B}_{\lambda, \Lambda}}(-\operatorname{Tr}(B . A))=-\Lambda \sum_{\mu_{i}(A)>0} \mu_{i}(A)-\lambda \sum_{\mu_{i}(A)<0} \mu_{i}(A) .
\end{aligned}
$$

The associated universal Riemannian operators $\mathcal{M}_{\lambda, \Lambda}^{ \pm}$are the so called Pucci's extremal operators, which are the prototype Hamilton-Jacobi-Bellman operators and, perhaps, the simplest example of Isaacs operators. They are proper, positively 1-homogeneous, Lipschitz continuous and uniformly elliptic with ellipticity constants $0<\lambda \leq \Lambda$. Moreover, it is well known that the uniform ellipticity of $F$ can be characterized by means of the extremal operators $\mathcal{M}_{\lambda, \Lambda}^{ \pm}$via

$$
\mathcal{M}_{\lambda, \Lambda}^{-}(x, Q) \leq F(x, s, q, Q)-F(x, s, q, 0) \leq \mathcal{M}_{\lambda, \Lambda}^{+}(x, Q) .
$$

(see e.g. [21, Lemma 2.2]). Furthermore, by [21, Lemma 2.10]

$$
\underline{\mathcal{M}}_{\lambda, \Lambda}^{-}\left(A-\alpha v \otimes v^{T}\right) \geq \underline{\mathcal{M}}_{\lambda, \Lambda}^{-}(A)+\alpha \lambda|v|^{2},
$$

we get the following

Lemma 3.4 Let $F: \widetilde{J}^{2} M \rightarrow \mathbb{R}$ be a proper operator. If $F$ is uniformly elliptic, then it is partially elliptic.

Proof Assume that $F$ is uniformly elliptic with ellipticity constants $0<\lambda \leq \Lambda$ and fix $x_{\mathrm{o}} \in M$. Then, for any $x \in B\left(x_{\mathrm{o}}, R\left(x_{\mathrm{o}}\right)\right)$ with $x \neq x_{\mathrm{o}}$, since $\left(\mathrm{d} f_{x_{\mathrm{o}}}\right)(x) \neq 0_{x}$, by (3.2) and (3.3) we get

$$
\begin{aligned}
F\left(x, 0,\left(\mathrm{~d} f_{x_{0}}\right)\right. & \left.(x),\left(D^{2} f_{x_{0}}\right)(x)-\alpha\left(\mathrm{d} f_{x_{0}}\right)(x) \otimes\left(\mathrm{d} f_{x_{0}}\right)(x)\right) \\
& \geq F\left(x, 0,\left(\mathrm{~d} f_{x_{0}}\right)(x), 0\right)+\mathcal{M}_{\lambda, \Lambda}^{-}\left(x,\left(D^{2} f_{x_{0}}\right)(x)-\alpha\left(\mathrm{d} f_{x_{0}}\right)(x) \otimes\left(\mathrm{d} f_{x_{0}}\right)(x)\right) \\
& \geq F\left(x, 0,\left(\mathrm{~d} f_{x_{0}}\right)(x), 0\right)+\mathcal{M}_{\lambda, \Lambda}^{-}\left(x,\left(D^{2} f_{x_{0}}\right)(x)\right)+\alpha \lambda\left|\left(\mathrm{d} f_{x_{0}}\right)(x)\right|^{2}
\end{aligned}
$$

and therefore $F$ is lower partially elliptic by taking $\alpha$ large enough. On the other hand, the operator

$$
F^{-}: \widetilde{J}^{2} M \rightarrow \mathbb{R}, \quad F^{-}(x, s, q, Q):=-F(x,-s,-q,-Q)
$$

is uniformly elliptic as well. Hence, by means of our previous computation, we obtain that $F^{-}$is lower partially elliptic, which is equivalent to say that $F$ is upper partially elliptic. 


\section{Pucci's Operators from [51]}

Fix a constant $0<\alpha \leq \frac{1}{n}$ and consider the set $\tilde{\mathcal{B}}_{\alpha}:=\{B \in \operatorname{Sym}(n): B \geq$ $\left.\alpha I_{n}, \operatorname{Tr}(B)=1\right\}$. Then, we define the functions

$$
\begin{aligned}
& \underline{\mathcal{P}}_{\alpha}^{+}: \operatorname{Sym}(n) \rightarrow \mathbb{R}, \quad \underline{\mathcal{P}}_{\alpha}^{+}(A):=\sup _{B \in \tilde{\mathcal{B}}_{\alpha}}(-\operatorname{Tr}(B . A))=-\alpha \operatorname{Tr}(A)-(1-n \alpha) \mu_{1}(A), \\
& \underline{\mathcal{P}}_{\alpha}^{-}: \operatorname{Sym}(n) \rightarrow \mathbb{R}, \quad \underline{\mathcal{P}}_{\alpha}^{-}(A):=\inf _{B \in \tilde{\mathcal{B}}_{\alpha}}(-\operatorname{Tr}(B . A))=-\alpha \operatorname{Tr}(A)-(1-n \alpha) \mu_{n}(A) .
\end{aligned}
$$

We call the associated universal Riemannian operators $\mathcal{P}_{\alpha}^{ \pm}$original Pucci's operators, as they were firstly introduced in the Euclidean case by Pucci in [51]. They are proper, positively 1-homogeneous, Lipschitz continuous and uniformly elliptic with ellipticity constants $0<\alpha \leq 1-(n-1) \alpha$, but do not allow to characterize uniform ellipticity of fully nonlinear operators as their extremal counterpart $\mathcal{M}_{\lambda, \Lambda}^{ \pm}$. Then, by means of (3.2), they are related with $\mathcal{M}_{\lambda, \Lambda}^{ \pm}$via

$$
\mathcal{M}_{\alpha, 1-(n-1) \alpha}^{-}(x, Q) \leq \mathcal{P}_{\alpha}^{-}(x, Q) \leq \mathcal{P}_{\alpha}^{+}(x, Q) \leq \mathcal{M}_{\alpha, 1-(n-1) \alpha}^{+}(x, Q)
$$

\section{p-Laplacian Operator}

Let $1<p<\infty$ and consider the function

$$
\underline{\mathcal{F}}_{p}:\left(\mathbb{R}^{n} \backslash\{0\}\right) \times \operatorname{Sym}(n) \rightarrow \mathbb{R}, \quad \underline{\mathcal{F}}_{p}(v, A):=-|v|^{p-2}\left(\operatorname{Tr}(A)+(p-2)|v|^{-2} v^{T} \cdot A \cdot v\right) .
$$

The associated universal Riemannian operator $\mathcal{F}_{p}$ is the so called $p$-Laplacian, which normally appears in divergence form as

$$
\mathcal{F}_{p}[u]=-\operatorname{div}\left(|\nabla u|^{p-2} \nabla u\right) .
$$

It is proper, continuous and positively $(p-1)$-homogeneous. Moreover, for any $v \neq 0$ we have

$$
\begin{aligned}
& \underline{\mathcal{F}}_{p}(v, c A)=c \underline{\mathcal{F}}_{p}(v, A) \quad \text { for any } c \in \mathbb{R}, \\
& \underline{\mathcal{F}}_{p}\left(v, A-\alpha v \otimes v^{T}\right)=\underline{\mathcal{F}}_{p}(v, A)+\alpha(p-1)|v|^{p}
\end{aligned}
$$

and so $\mathcal{F}_{p}$ is partially elliptic, even though it is not uniformly elliptic.

We conclude by saying that the 1 -homogeneous version of the $p$-Laplacian $\mathcal{F}_{p}$, called game-theoretic $p$-Laplacian, that is the operator $\mathcal{F}_{p}^{G}$ associated to

$$
\underline{\mathcal{F}}_{p}^{G}(v, A):=|v|^{2-p} \underline{\mathcal{F}}_{p}(v, A)=-\operatorname{Tr}(A)-(p-2)|v|^{-2} v^{T} \cdot A \cdot v .
$$

is proper, continuous and positively 1-homogeneous. Moreover, it is easy to check that it is partially elliptic via condition (ii) in Sect. 3.1. 


\section{$\infty$-Laplacian Operator}

Consider the function

$$
\underline{\mathcal{F}}_{\infty}: \mathbb{R}^{n} \oplus \operatorname{Sym}(n) \rightarrow \mathbb{R}, \quad \underline{\mathcal{F}}_{\infty}(v, A):=-v^{T} . A . v .
$$

The associated universal Riemannian operators $\mathcal{F}_{\infty}$ is the so called $\infty$-Laplacian, which is proper, continuous and positively 3-homogeneous. Since

$$
\begin{aligned}
& \underline{\mathcal{F}}_{\infty}(v, c A)=c \underline{\mathcal{F}}_{\infty}(v, A) \quad \text { for any } c \in \mathbb{R}, \\
& \underline{\mathcal{F}}_{\infty}\left(v, A-\alpha v \otimes v^{T}\right)=\underline{\mathcal{F}}_{\infty}(v, A)+\alpha|v|^{4},
\end{aligned}
$$

we conclude that $\mathcal{F}_{\infty}$ is partially elliptic. Similarly, the positively $h$-homogeneous version $\mathcal{F}_{\infty}^{h}$ of the $\infty$-Laplacian, which is the operator associated to

$$
\underline{\mathcal{F}}_{\infty}^{h}(v, A):=|v|^{h-3} \underline{\mathcal{F}}_{\infty}(v, A)=-|v|^{h-3} v^{T} \cdot A \cdot v,
$$

is proper, continuous and it is easy to check that it is partially elliptic.

\section{Mean Curvature Operator}

Consider the function

$$
\underline{\mathcal{F}}_{\mathrm{mc}}: \mathbb{R}^{n} \oplus \operatorname{Sym}(n) \rightarrow \mathbb{R}, \quad \underline{\mathcal{F}}_{\mathrm{mc}}(v, A):=\left(1+|v|^{2}\right)^{-\frac{3}{2}}\left(-\left(1+|v|^{2}\right) \operatorname{Tr}(A)+v^{T} \cdot A \cdot v\right) .
$$

The associated universal Riemannian operator $\mathcal{F}_{\mathrm{mc}}$ is the so called mean curvature operator, which can be written in divergence form as

$$
\mathcal{F}_{\mathrm{mc}}[u]=-\operatorname{div}\left(\frac{\nabla u}{\sqrt{1+|\nabla u|^{2}}}\right) .
$$

It is proper and continuous. Moreover, since

$$
\begin{aligned}
& \underline{\mathcal{F}}_{\mathrm{mc}}(v, c A)=c \underline{\mathcal{F}}_{\mathrm{mc}}(v, A) \quad \text { for any } c \in \mathbb{R}, \\
& \underline{\mathcal{F}}_{\mathrm{mc}}\left(v, A-\alpha v \otimes v^{T}\right)=\underline{\mathcal{F}}_{\mathrm{mc}}(v, A)+\alpha|v|^{2}\left(1+|v|^{2}\right)^{-\frac{3}{2}},
\end{aligned}
$$

it follows that $\mathcal{F}_{\mathrm{mc}}$ is partially elliptic.

\section{The Strong Maximum Principle}

Let $(M, g)$ be a Riemannian manifold. First, we are going to prove a technical result which asserts the propagation of maxima on small balls for subsolutions to lower partially elliptic operators on $(M, g)$. Namely 
Proposition 4.1 Let $F: \widetilde{J}^{2} M \rightarrow \mathbb{R}$ be a proper, lower semicontinuous operator which is lower partially elliptic (l.p.e.) and has the lower scaling property (l.s.p.). Let $\Omega \subset M$ be a domain and $u \in \operatorname{USC}(\Omega)$ be a viscosity subsolution to $F[u]=0$ on $\Omega$ which attains a nonnegative maximum at some point $x_{\mathrm{O}} \in \Omega$. Then, for any radius $0<r<\frac{1}{2} \min \left\{R\left(x_{0}\right)\right.$, dist $\left.\left(x_{0}, \partial \Omega\right)\right\}$ and for any point $y \in S\left(x_{0}, r\right)$, the ball $B(y, r)$ contains a point $\tilde{x}=\tilde{x}(y, r)$ with $u(\tilde{x})=u\left(x_{0}\right)$.

Proof Assume by contradiction that there exist a finite radius $0<r_{\mathrm{O}}<$ $\frac{1}{2} \min \left\{R\left(x_{\mathrm{o}}\right)\right.$, dist $\left.\left(x_{\mathrm{o}}, \partial \Omega\right)\right\}$ and a point $y_{\mathrm{o}} \in S\left(x_{\mathrm{o}}, r_{\mathrm{o}}\right)$ such that $u(x)<u\left(x_{\mathrm{o}}\right)$ for any $x \in B\left(y_{0}, r_{0}\right)$. Let $\alpha>0$ to be determined, $f_{y_{0}}(x)=\frac{1}{2} \operatorname{dist}\left(x, y_{0}\right)^{2}$ as in (2.1) and consider the function

$$
h: B\left(y_{\mathrm{o}}, R\left(y_{\mathrm{o}}\right)\right) \rightarrow \mathbb{R}, \quad h(x):=-\exp \left(-\alpha f_{y_{\mathrm{o}}}(x)\right)+\exp \left(-\alpha \frac{r_{\mathrm{o}}^{2}}{2}\right) .
$$

Notice that, since $x \mapsto R(x)$ is 1-Lipschitz, it follows that $r_{\mathrm{O}}<R\left(y_{\mathrm{o}}\right)$. Indeed (see Sect. 2):

- if $R\left(x_{\mathrm{o}}\right)=+\infty$, then $R\left(y_{\mathrm{o}}\right)=+\infty$ as well,

- if $R\left(x_{\mathrm{o}}\right)<+\infty$, then $R\left(y_{\mathrm{o}}\right)<+\infty$ and $R\left(y_{\mathrm{o}}\right) \geq R\left(x_{\mathrm{o}}\right)-r_{\mathrm{o}}>\frac{1}{2} R\left(x_{\mathrm{o}}\right)>r_{\mathrm{o}}$.

Moreover, from the very definition, it comes that $h(x)=0$ for any $x \in S\left(y_{0}, r_{0}\right)$ and $-1<h(x)<0$ for any $x \in B\left(y_{\mathrm{o}}, r_{\mathrm{o}}\right)$. Letting $c:=\alpha \exp \left(-\alpha \frac{r_{\mathrm{o}}^{2}}{2}\right)>0$, we get

$$
\begin{aligned}
& h\left(x_{\mathrm{o}}\right)=0, \quad(\mathrm{~d} h)\left(x_{\mathrm{o}}\right)=c\left(\mathrm{~d} f_{y_{\mathrm{o}}}\right)\left(x_{\mathrm{o}}\right) \\
& \quad\left(D^{2} h\right)\left(x_{\mathrm{o}}\right)=c\left(\left(D^{2} f_{y_{\mathrm{o}}}\right)\left(x_{\mathrm{o}}\right)-\alpha\left(\mathrm{d} f_{y_{\mathrm{o}}}\right)\left(x_{\mathrm{o}}\right) \otimes\left(\mathrm{d} f_{y_{\mathrm{o}}}\right)\left(x_{\mathrm{o}}\right)\right) .
\end{aligned}
$$

Hence, by the lower partial ellipticity and the lower scaling property, if we choose $\alpha>0$ big enough, we get $0<c<1$ and

$$
\begin{aligned}
& F\left(x_{\mathrm{o}}, h\left(x_{\mathrm{o}}\right),(\mathrm{d} h)\left(x_{\mathrm{o}}\right),\left(D^{2} h\right)\left(x_{\mathrm{o}}\right)\right) \\
& \geq \eta_{y_{\mathrm{o}}}(c) F\left(x_{\mathrm{o}}, 0,\left(\mathrm{~d} f_{y_{\mathrm{o}}}\right)\left(x_{\mathrm{o}}\right),\left(D^{2} f_{y_{\mathrm{o}}}\right)\left(x_{\mathrm{o}}\right)-\alpha\left(\mathrm{d} f_{y_{\mathrm{o}}}\right)\left(x_{\mathrm{o}}\right) \otimes\left(\mathrm{d} f_{y_{\mathrm{o}}}\right)\left(x_{\mathrm{o}}\right)\right)>0 .
\end{aligned}
$$

By the lower semicontinuity of $F$, there exist two constants $0<r<\min \left\{r_{\mathrm{o}}, R\left(y_{\mathrm{o}}\right)-\right.$ $\left.r_{\mathrm{o}}\right\}$ and $C>0$ such that $F[h](x) \geq C$ for any $x \in U:=B\left(x_{\mathrm{o}}, r\right) \cap B\left(y_{\mathrm{o}}, r_{\mathrm{o}}\right)$. By the lower scaling property, the function $\varepsilon h$ is a strict supersolution of $F[u]=0$ on $U$ for any $0<\varepsilon<1$. Assume also that $u(x)-u\left(x_{0}\right) \leq 0$ for any $x \in B\left(x_{0}, r\right)$ and recall that $u(x)-u\left(x_{\mathrm{o}}\right)<0$ for any $x \in B\left(y_{\mathrm{o}}, r_{\mathrm{o}}\right)$. Then, since $h(x)<0$ for any $x \in \partial U \cap B\left(y_{0}, r_{\mathrm{o}}\right)$ and $h(x)=0$ for any $x \in \partial U \cap S\left(y_{0}, r_{\mathrm{o}}\right)$, we can choose $0<\varepsilon_{\mathrm{o}}<1$ in such a way that $u(x)-u\left(x_{\mathrm{o}}\right) \leq \varepsilon_{\mathrm{o}} h(x)$ for any $x \in \partial U$. Let us define then

$$
\psi: \bar{B}\left(x_{\mathrm{o}}, r\right) \rightarrow \mathbb{R}, \quad \psi(x):=u(x)-\left(u\left(x_{\mathrm{o}}\right)+\varepsilon_{\mathrm{o}} h(x)\right)
$$

We claim that $\psi(x) \leq 0$ for any $x \in \bar{U} \subset \bar{B}\left(x_{0}, r\right)$. In fact, assuming by contradiction that $\max _{x \in \bar{U}} \psi(x)>0$, then there exists $x^{\prime} \in U$ such that $\psi\left(x^{\prime}\right)=\max _{x \in \bar{U}} \psi(x)>0$. 
But then, since $u\left(x_{\mathrm{o}}\right)+\varepsilon_{\mathrm{o}} h$ is of class $\mathcal{C}^{2}, \mathrm{~d} h\left(x^{\prime}\right) \neq 0$ and $u\left(x_{\mathrm{o}}\right) \geq 0$, owing also to the fact that $F$ is proper, we get

$$
F\left(x^{\prime}, \varepsilon_{\mathrm{o}} h\left(x^{\prime}\right), \varepsilon_{\mathrm{o}} \mathrm{d} h\left(x^{\prime}\right), \varepsilon_{\mathrm{o}} D^{2} h\left(x^{\prime}\right)\right) \leq F\left(x^{\prime}, u\left(x^{\prime}\right), \varepsilon_{\mathrm{o}} \mathrm{d} h\left(x^{\prime}\right), \varepsilon_{\mathrm{o}} D^{2} h\left(x^{\prime}\right)\right) \leq 0,
$$

which contradicts our previous claim. By construction, this implies that $\psi \leq 0$ in $\bar{B}\left(x_{\mathrm{o}}, r\right)$. Since $\psi\left(x_{\mathrm{o}}\right)=0$, together with the fact that $u$ is a viscosity subsolution, we get

$$
F\left(x_{\mathrm{o}}, \varepsilon_{\mathrm{o}} h\left(x_{\mathrm{o}}\right), \varepsilon_{\mathrm{o}} \mathrm{d} h\left(x_{\mathrm{o}}\right), \varepsilon_{\mathrm{o}} D^{2} h\left(x_{\mathrm{o}}\right)\right) \leq F\left(x_{\mathrm{o}}, u\left(x_{\mathrm{o}}\right), \varepsilon_{\mathrm{o}} \mathrm{d} h\left(x_{\mathrm{o}}\right), \varepsilon_{\mathrm{o}} D^{2} h\left(x_{\mathrm{o}}\right)\right) \leq 0,
$$

a contradiction with (4.1).

As a direct corollary, we obtain the following version of the Strong Maximum Principle ((SMP) for short).

Theorem 4.2 (Strong Maximum Principle) Let $F: \widetilde{J}^{2} M \rightarrow \mathbb{R}$ be a proper, lower semicontinuous operator which is lower partially elliptic and has the lower scaling property. Let $\Omega \subset M$ be a domain and $u \in \operatorname{USC}(\Omega)$ be a viscosity subsolution to $F[u]=0$ on $\Omega$. If $u$ achieves a nonnegative maximum in $\Omega$, then $u$ is constant.

Proof Let $\tilde{x} \in \Omega$ be a maximum point for $u$ in $\Omega$ and set $L:=u(\tilde{x})>0, K:=\{x \in$ $\Omega: u(x)=L\}$. Assume by contradiction that $K \subsetneq \Omega$. Since $u$ is upper semicontinuous and $\tilde{x}$ is a maximum point, it follows that $K=\{x \in \Omega: u(x) \geq L\}$ and so it is closed in $\Omega$. Then, by classical arguments (see e.g. [31]) we can find a point $y_{\mathrm{o}} \in \Omega \backslash K$ such that $\operatorname{dist}\left(y_{0}, \partial \Omega\right)>\operatorname{dist}\left(y_{0}, \partial K\right)=r$. This means that there exists $x_{\mathrm{o}} \in S\left(y_{0}, r\right)$ such that $u\left(x_{\mathrm{o}}\right)=L$, while $u(x)<L$ for any $x \in B\left(y_{0}, r\right)$. However, this is in contradiction with Proposition 4.1.

Remark 4.3 If the Riemannian manifold $(M, g)$ has nonnegative sectional curvature, then one can replace the condition (1.p.e.) in Theorem 4.2 with

(1.p.e.') there exists a function $\alpha_{\mathrm{o}}: M \rightarrow[0,+\infty)$ such that

$$
F\left(x, 0, q, g_{x}-\alpha q \otimes q\right)>0 \quad \text { for any } x \in M, q \in T_{x}^{*} M \backslash\{0\}, \alpha>\alpha_{\mathrm{o}}(x) \text {. }
$$

Indeed, under the hypothesis of nonnegative sectional curvature, by the Rauch Comparison Theorem it follows that $\left(D^{2} f_{x_{0}}\right)(x) \leq g_{x}$ for any $x_{\mathrm{o}} \in M$ and $x \in B\left(x_{\mathrm{o}}, \operatorname{inj}\left(x_{\mathrm{o}}\right)\right)$ (see e.g. [20, Lemma 3.1]). This is the same non-totally degeneracy condition found in the Euclidean case by Bardi-Da Lio [7].

Counterexample 4.4 (From [10, Example 3.17] and [39]) Assume that $(M, g)$ has nonnegative sectional curvature and fix a point $x_{\mathrm{o}} \in M$. Let us consider the nonlinear PDE

$$
-\frac{\Delta u}{1+|\Delta u|}+f(x)=0
$$


where $f: M \rightarrow \mathbb{R}$ is defined by setting $f\left(x_{0}\right):=-1$ and $f(x):=0$ for any $x \in M \backslash\left\{x_{\mathrm{o}}\right\}$. It is straightforward to check that this operator is proper, but the scaling condition and (l.p.e.') fail at $x_{\mathrm{o}}$. In fact, the (SMP) is violated by the viscosity subsolution

$$
u: M \rightarrow \mathbb{R}, \quad u(x)=\left\{\begin{array}{ll}
0 & \text { if } x \neq x_{\mathrm{o}} \\
1 & \text { if } x=x_{\mathrm{o}}
\end{array} .\right.
$$

We stress now that part of the proof of Proposition 4.1 can be adapted to prove a viscosity version of the Hopf boundary Lemma (see [24, Appx E] and [31, Lemma 3.4] for the corresponding statements in the linear case). Before doing that, we recall that:

(i.b.c.) a domain $\Omega \subset M$ satisfies the interior ball condition at a boundary point $x_{\mathrm{o}} \in \partial \Omega$ if there exist $y \in \Omega$ and $0<r<R(y)$ such that $B(y, r) \subset \Omega$ and $S(y, r) \cap \partial \Omega=\left\{x_{0}\right\}$.

Under such hypothesis, for any point $x \in B(y, r)$ there exists a unique unit speed minimizing geodesic $\gamma_{x}:\left[-\delta_{x}, 0\right) \rightarrow B(y, r)$ such that $\gamma_{x}\left(-\delta_{x}\right)=x$ and $\gamma_{x}(0):=$ $\lim _{t \rightarrow 0^{+}} \gamma_{x}(-t)=x_{0}$.

Theorem 4.5 (Hopf boundary Lemma) Let $F: \widetilde{J}^{2} M \rightarrow \mathbb{R}$ be a proper, lower semicontinuous operator which is lower partially elliptic and has the lower scaling property. Let $\Omega \subset M$ be a domain and $u \in \operatorname{USC}(\Omega)$ be a subsolution to $F[u]=0$ on $\Omega$. Assume that $\Omega$ satisfies the interior ball condition at some boundary point $x_{0} \in \partial \Omega$ and set $u\left(x_{\mathrm{o}}\right):=\lim _{r \rightarrow 0^{+}} \sup \left\{u(x): x \in \Omega \cap B\left(x_{\mathrm{o}}, r\right)\right\}$. If $0 \leq u\left(x_{\mathrm{o}}\right)<+\infty$ and $u<u\left(x_{\mathrm{o}}\right)$ in $\Omega$, then for any interior ball $B(y, r) \subset \Omega$ as in (i.b.c.) it holds

$$
\liminf _{t \rightarrow 0^{+}} \frac{\left(u \circ \gamma_{x}\right)(0)-\left(u \circ \gamma_{x}\right)(-t)}{t}>0 \quad \text { for any } x \in B\left(y_{0}, r_{0}\right)
$$

where $\gamma_{x}$ is the unique geodesic as above.

Proof Let $B(y, r) \subset \Omega$ be as in (i.b.c.). Let also $\alpha>0, f_{y}(x)=\frac{1}{2} \operatorname{dist}(x, y)^{2}$ as in (2.1) and consider the function

$$
h: B(y, R(y)) \rightarrow \mathbb{R}, \quad h(x):=-\exp \left(-\alpha f_{y}(x)\right)+\exp \left(-\alpha \frac{r^{2}}{2}\right) .
$$

By repeating the same argument as in the proof of Proposition 4.1, one can choose $\alpha>0$ big enough, $0<\tilde{r}<R(y)-r$ small enough and $0<\varepsilon<1$ small enough such that $u(x) \leq u\left(x_{\mathrm{o}}\right)+\varepsilon h(x)$ for any $x \in \bar{U}$, with $U:=B\left(x_{\mathrm{o}}, \tilde{r}\right) \cap B(y, r)$. Then, setting $c:=\alpha \exp \left(-\alpha \frac{r^{2}}{2}\right)$ we get

$$
\begin{aligned}
& \liminf _{t \rightarrow 0^{+}} \frac{\left(u \circ \gamma_{x}\right)(0)-\left(u \circ \gamma_{x}\right)(-t)}{t} \\
& \geq \varepsilon \mathrm{d} h\left(x_{\mathrm{o}}\right)\left(\dot{\gamma}_{x}(0)\right)=\varepsilon c g_{x_{\mathrm{o}}}\left(\left(\nabla f_{y}\right)\left(x_{\mathrm{o}}\right), \dot{\gamma}_{x}(0)\right)>0
\end{aligned}
$$

which concludes the proof. 
Remark 4.6 In the hypotheses of Theorem 4.5, if $u \in \mathcal{C}^{1}(\bar{\Omega})$, then (4.2) reads as

$$
\mathrm{d} u\left(x_{\mathrm{o}}\right)(v)>0 \quad \text { for any } v \in T_{x_{0}} M \text { such that } g_{x_{0}}\left(\left(\nabla f_{y}\right)\left(x_{\mathrm{o}}\right), v\right)>0,
$$

which is the classical statement of the Hopf Boundary Lemma (see [31, Lemma 3.4] and [24, Appx E]).

Finally, we remark that Theorem 4.2 can be used to derive a version of the Strong Minimum Principle ((SmP) for short). Indeed

Theorem 4.7 (Strong Minimum Principle) Let $F: \widetilde{J}^{2} M \rightarrow \mathbb{R}$ be a proper, upper semicontinuous operator which is upper partially elliptic (u.p.e.) and has the upper scaling property (u.s.p.). Let $\Omega \subset M$ be a domain and $v \in \operatorname{LSC}(\Omega)$ be a viscosity supersolution to $F[u]=0$ on $\Omega$. If $v$ achieves a nonpositive minimum in $\Omega$, then $v$ is constant.

Proof Given such $F: \widetilde{J}^{2} M \rightarrow \mathbb{R}$ and $v \in \operatorname{LSC}(\Omega)$, it is straightforward to realize that $u:=-v$ is a viscosity subsolution to the equation $F^{-}[u]=0$, with

$$
F^{-}: \widetilde{J}^{2} M \rightarrow \mathbb{R}, \quad F^{-}(x, s, q, Q):=-F(x,-s,-q,-Q) .
$$

Since $F^{-}$verifies all the hypothesis of Theorem 4.2, we get the thesis.

Remark 4.8 The proof of Proposition 4.1, Theorems 4.2 and 4.7 remain untouched if we replace the conditions (l.s.p.) and (u.s.p.), respectively, with the following:

(l.s.p.') there exists $\hat{F}$ satisfying (1.s.p.) and, for any $x_{0} \in M$, a third function $\hat{\eta}_{x_{0}}$ : $(0,1) \rightarrow \mathbb{R}$ such that $\lim _{c \rightarrow 0^{+}} \hat{\eta}_{x_{0}}(c)=0$ and

$$
\begin{aligned}
& F\left(x, c s, c\left(\mathrm{~d} f_{x_{\mathrm{o}}}\right)(x), c\left(\left(D^{2} f_{x_{\mathrm{o}}}\right)(x)-\alpha\left(\mathrm{d} f_{x_{\mathrm{o}}}\right)(x) \otimes\left(\mathrm{d} f_{x_{\mathrm{o}}}\right)(x)\right)\right) \geq \\
& \quad \hat{F}\left(x, c s, c\left(\mathrm{~d} f_{x_{\mathrm{o}}}\right)(x), c\left(\left(D^{2} f_{x_{\mathrm{o}}}\right)(x)-\alpha\left(\mathrm{d} f_{x_{\mathrm{o}}}\right)(x) \otimes\left(\mathrm{d} f_{x_{\mathrm{o}}}\right)(x)\right)\right)+\eta_{x_{\mathrm{o}}}(c) \hat{\eta}_{x_{\mathrm{o}}}(c)
\end{aligned}
$$

for any $c \in(0,1], s \in[-1,0]$ and $x \in B\left(x_{0}, R\left(x_{0}\right)\right)$ with $x \neq x_{0}$;

(u.s.p.') there exists $\hat{F}$ satisfying (u.s.p.) and, for any $x_{\mathrm{o}} \in M$, a third function $\hat{\eta}_{x_{\mathrm{o}}}$ : $(0,1) \rightarrow \mathbb{R}$ such that $\lim _{c \rightarrow 0^{+}} \hat{\eta}_{x_{0}}(c)=0$ and

$$
\begin{aligned}
& F\left(x, c s, c\left(\mathrm{~d} f_{x_{\mathrm{o}}}\right)(x), c\left(\left(D^{2} f_{x_{\mathrm{o}}}\right)(x)-\alpha\left(\mathrm{d} f_{x_{\mathrm{o}}}\right)(x) \otimes\left(\mathrm{d} f_{x_{\mathrm{o}}}\right)(x)\right)\right) \leq \\
& \quad \hat{F}\left(x, c s, c\left(\mathrm{~d} f_{x_{\mathrm{o}}}\right)(x), c\left(\left(D^{2} f_{x_{\mathrm{o}}}\right)(x)-\alpha\left(\mathrm{d} f_{x_{\mathrm{o}}}\right)(x) \otimes\left(\mathrm{d} f_{x_{\mathrm{o}}}\right)(x)\right)\right)+\eta_{x_{\mathrm{o}}}(c) \hat{\eta}_{x_{\mathrm{o}}}(c)
\end{aligned}
$$

for any $c \in(0,1], s \in[0,1]$ and $x \in B\left(x_{0}, R\left(x_{0}\right)\right)$ with $x \neq x_{0}$.

We are going to list below some examples that fulfill the hypotheses of our (SMP) and $(\mathrm{SmP})$.

Example 4.9 All the universal Riemannian operators listed in Sect. 3.2, with the exception of the mean curvature operator and the Monge-Ampère operator (cf Remark 3.3), 
satisfy all the hypotheses of Theorems 4.2 and 4.7. Hence, both (SMP) and (SmP) are satisfied.

Actually, we can cover more general universal Riemannian operators, e.g. we can consider

$$
\underline{\mathcal{F}}: \mathbb{R}^{n} \oplus \operatorname{Sym}(n) \rightarrow \mathbb{R}, \quad \underline{\mathcal{F}}(v, A):=|v|^{\beta} \underline{\mathcal{M}}_{\lambda, \Lambda}^{ \pm}(A) \quad \text { with } \beta>1 .
$$

Then, the operator $\mathcal{F}$ associated to $\underline{\mathcal{F}}$, which has been studied in [15], is proper and positively $(\beta+1)$-homogeneous. Moreover, by [21, Lemma 2.10] we get

$$
\begin{aligned}
& \underline{\mathcal{F}}\left(v, A-\alpha v \otimes v^{T}\right) \geq|v|^{\beta} \underline{\mathcal{M}}_{\lambda, \Lambda}^{-}(A)+\alpha \lambda|v|^{2+\beta}, \\
& \underline{\mathcal{F}}\left(v, \alpha v \otimes v^{T}-A\right) \leq-|v|^{\beta} \underline{\mathcal{M}}_{\lambda, \Lambda}^{-}(A)-\alpha \lambda|v|^{2+\beta}
\end{aligned}
$$

and thus it is partially elliptic. Hence, both the (SMP) and the (SmP) hold true. This would extend [15, Proposition 2.15] to Riemannian manifolds.

Remark 4.10 To the best of our knowledge, this is the first (SMP) for viscosity solutions of the $p$-Laplace equation on Riemannian manifolds. Recently, some results have been established in [14] for $\mathcal{C}^{1}$ distributional solutions of equations driven by the $p$-Laplacian on Riemannian manifolds. However, while in the Euclidean case weak (distributional) and viscosity solutions agree (see [38]), we are not aware of a similar result in the more general setting of Riemannian manifolds.

Example 4.11 We stress that neither (1.s.p.) nor (u.s.p.) hold true for the mean curvature operator $\mathcal{F}_{\mathrm{mc}}$, but still it fulfills both (l.s.p.'), (u.s.p.') and so one can obtain the (SMP) and the (SmP) in virtue of Remark 4.8 (see also [7, Sec 3]). The same is true for the capillary surface equation

$$
n H u\left(1+|\nabla u|^{2}\right)^{\frac{3}{2}}-\left(1+|\nabla u|^{2}\right) \Delta u+D^{2} u(\nabla u, \nabla u)=0, \quad \text { with } H \in \mathbb{R} .
$$

Let us notice that the (SMP) for the mean curvature equation on Riemannian manifolds has been established recently in [14], while that for the capillary equation appears to be new.

Example 4.12 Let $\mathcal{F}=\mathcal{F}(x, q, Q)$ be a positively $h$-homogeneous universal Riemannian operator, with $h \in \mathbb{R}$. Let $a, c: M \rightarrow \mathbb{R}$ be two continuous functions such that $c(x) \geq 0, a(x)>0$ for any $x \in M$. Pick a number $k>0$ and assume that either $c \equiv 0$, or $k \geq h$. Then, the operator

$$
F(x, s, q, Q):=a(x) \mathcal{F}(x, q, Q)+c(x)|s|^{k-1} s
$$

verifies both the (SMP) and the (SmP). We stress that the capillarity equation (4.3) is of this form with $\mathcal{F}=\mathcal{F}_{\mathrm{mc}}, k=1, a \equiv 1$ and $c \equiv n H$.

In the special case when $\mathcal{F}=-\Delta_{p}$, which is homogeneous of degree $h=p-1$, the threshold $k \geq p-1$ has been found to be sharp in [57], cf also [7, Example 1] and the references therein. 
Example 4.13 Let $\mathcal{F}$ be a Hessian operator, that is a universal Riemannian operator associated to an Euclidean operator $\mathcal{F}: \operatorname{Sym}(n) \rightarrow \mathbb{R}$. Assume that $\mathcal{F}$ is continuous, uniformly elliptic and positively 1-homogeneous. Let also $b \in \mathcal{C}^{0}(M, T M)$ a continuous and bounded vector field on $M$. Then, the operators analyzed in [41] of the general form

$$
F[u]:=|\nabla u|^{p-2}(\mathcal{F}[u]+g(\nabla u, b))
$$

verify both the (SMP) and the (SmP).

\section{An Application: The Strong Comparison Principle}

Let $(M, g)$ be a Riemannian manifold. In this section, we are going to establish some consequences of the Strong Maximum Principle.

In the case of linear equations, being the difference of two solutions a solution as well, it turns out that the (SCP) is equivalent to the (SMP). In the nonlinear case, it is worth observing that the (SCP) implies the (SMP) whenever a constant solves the equation. However, the converse may not hold. To have a guideline for the general case, we begin with the following

Lemma 5.1 Let $\Omega \subset M$ be a domain and $0<\lambda \leq \Lambda$ be two constants. Consider a viscosity subsolution $u \in \operatorname{USC}(\Omega)$ and a classical supersolution $v \in \mathcal{C}^{2}(\Omega, \mathbb{R})$ of $\mathcal{M}_{\lambda, \Lambda}^{-}[u]=0$ on $\Omega$ satisfying $u \leq v$ in $\Omega$ and assume there exists $x_{\mathrm{o}} \in \Omega$ such that $u\left(x_{\mathrm{o}}\right)=v\left(x_{\mathrm{o}}\right)$. Then, $u \equiv v$ in $\Omega$.

Proof It is sufficient to prove that $w:=u-v$ is a subsolution of $\mathcal{M}_{\lambda, \Lambda}^{-}[u]=0$ on $\Omega$ and then the conclusion follows by Theorem 4.2. Indeed, since $w \leq 0$ by assumptions, if there exists $x_{\mathrm{o}} \in \Omega$ such that $w\left(x_{\mathrm{o}}\right)=0$, then the (SMP) would imply $w=0$ on the whole $\Omega$.

Let us prove now that $w$ is a subsolution to $\mathcal{M}_{\lambda, \Lambda}^{-}[u]=0$ following [21, Lemma $2.12]$. Let $\psi \in \mathcal{C}^{2}(\Omega, \mathbb{R})$ be such that $w-\psi \leq 0$ around $x_{\mathrm{o}}$ and $w\left(x_{\mathrm{o}}\right)=\psi\left(x_{\mathrm{o}}\right)$. Since $u$ is a viscosity subsolution, we get

$$
\mathcal{M}_{\lambda, \Lambda}^{-}[\psi+v]\left(x_{\mathrm{o}}\right) \leq 0
$$

Moreover, being $\mathcal{M}_{\lambda, \Lambda}^{-}$sub-additive (see e.g. [21, Lemma 2.10]), it holds that

$$
\mathcal{M}_{\lambda, \Lambda}^{-}[\psi]\left(x_{\mathrm{o}}\right)+\mathcal{M}_{\lambda, \Lambda}^{-}[v]\left(x_{\mathrm{o}}\right) \leq 0
$$

Since $\mathcal{M}_{\lambda, \Lambda}^{-}[v]\left(x_{0}\right) \geq 0$ in classical sense by hypothesis, we conclude that $\mathcal{M}_{\lambda, \Lambda}^{-}[\psi]\left(x_{\mathrm{o}}\right) \leq 0$ as desired.

Remark 5.2 Let us point out that the proof of Lemma 5.1 still holds true for any continuous, uniformly elliptic operator $F: J^{2} M \rightarrow \mathbb{R}$ which is sub-additive, i.e. satisfying $F\left[\varphi_{1}-\varphi_{2}\right] \leq F\left[\varphi_{1}\right]-F\left[\varphi_{2}\right]$ for any pair of $\mathcal{C}^{2}$ functions $\varphi_{1}, \varphi_{2}$. Examples of 
such operators are the Pucci's extremal operators and, more in general, the HamiltonJacobi-Bellman operators (see [6]).

Let us stress now that handling viscosity solutions requires more care and the use of the strong and weak maximum principles, as in $[10,36]$. Indeed, the fact that $w=u-v$ is a viscosity subsolution to the initial equation is not straightforward in general (see e.g. [21, Thm 5.3] for the uniformly elliptic case). Below, we prove the (SCP) by combining the (SMP) in Theorem 4.2 and the weak comparison principle in [4, Cor 4.8] following the same lines of [36, Thm 5.3 and Prop 5.5].

Before doing that, given a proper and continuous operator $F: J^{2} M \rightarrow \mathbb{R}$, we recall the following two properties:

(i.u.c.) $F$ is intrinsically uniformly continuous with respect to $x$ if there exists a modulus of continuity $\omega$ such that

$$
\left|F(y, s, q, Q)-F\left(x, s, L_{x y}^{*} q, L_{x y}^{*} Q\right)\right| \leq \omega(\operatorname{dist}(x, y))
$$

for any $x, y \in M$ with $\operatorname{dist}(x, y)<\min \{\operatorname{inj}(x), \operatorname{inj}(y)\}$ and $(s, q, Q) \in$ $J_{y}^{2} M$

(u.l.p.) $F$ satisfies the uniform Lipschitz condition if there exists $C>0$ such that

$$
\left|F\left(x, s_{1}, q_{1}, Q\right)-F\left(x, s_{2}, q_{2}, Q\right)\right| \leq C\left(\left|s_{1}-s_{2}\right|+\left|q_{1}-q_{2}\right|\right)
$$

for any $x \in M$ and $\left(s_{1}, q_{1}, Q\right),\left(s_{2}, q_{2}, Q\right) \in J_{x}^{2} M$.

Remark 5.3 Any universal Riemannian operator $\mathcal{F}$ verifies (5.1) with $\omega=0$. Indeed, the $\mathrm{O}(n)$-invariance implies that the corresponding Euclidean operator is of the form $\underline{\mathcal{F}}(s, v, A)=f(s,|v|$, eigenvalues ofA $)$. Then, since $\left|L_{x y}^{*} q\right|=|q|$ and $Q, L_{x y}^{*} Q$ have the same eigenvalues, condition (i.u.c.) is fulfilled.

Then, the result reads as follows.

Theorem 5.4 Assume that $(M, g)$ has nonnegative sectional curvature and let $\Omega \subset M$ be a domain. Let $F: J^{2} M \rightarrow \mathbb{R}$ be a continuous operator which is uniformly elliptic and satisfying both (i.u.c.), (u.l.p.). Consider a viscosity subsolution $u \in \mathrm{USC}(\Omega)$ and a viscosity supersolution $v \in \operatorname{LSC}(\Omega)$ of $F[u]=0$ on $\Omega$ satisfying $u \leq v$ in $\Omega$ and assume there exists $x_{\mathrm{o}} \in \Omega$ such that $u\left(x_{\mathrm{o}}\right)=v\left(x_{\mathrm{o}}\right)$. Then, $u \equiv v$ in $\Omega$.

Before going ahead with the proof of Theorem 5.4, we introduce the linearized operator 


$$
\begin{aligned}
\Delta_{F}: J^{2} M \rightarrow \mathbb{R}, \quad \Delta_{F}(x, s, q, Q)= & \inf \{F(x, s+\tilde{s}, q+\tilde{q}, Q+\tilde{Q}) \\
& \left.-F(x, s, q, Q):(\tilde{s}, \tilde{q}, \tilde{Q}) \in J_{x}^{2} M\right\}
\end{aligned}
$$

and its lower semicontinuous envelope $\left(\Delta_{F}\right)_{*}$, which is defined by

$$
\begin{aligned}
\left(\Delta_{F}\right)_{*}(x, s, q, Q)= & \lim _{r \rightarrow 0^{+}}\left(\operatorname { i n f } \left\{\Delta_{F}\left(y, s^{\prime}, q^{\prime}, Q^{\prime}\right): \operatorname{dist}(x, y)\right.\right. \\
& \left.\left.+\left|s-s^{\prime}\right|+\left|q-L_{x y}^{*} q^{\prime}\right|+\left|Q-L_{x y}^{*} Q^{\prime}\right|<r\right\}\right) .
\end{aligned}
$$

Then, we will need the following

Lemma 5.5 Assume the same hypothesis of Theorem 5.4 and set $w:=u-v$. Then, $w$ is a viscosity subsolution of $\left(\Delta_{F}\right)_{*}[u]=0$.

Notice that Lemma 5.5 can be obtained by following the proof of [36, Prop 5.5], which is stated in the Euclidean case $(M, g)=\left(\mathbb{R}^{n},\langle\rangle,\right)$. However, for convenience of the reader, we provide a proof of this result by following the authors' original approach.

Proof of Lemma 5.5 Arguing by contradiction, we assume that there exist a function $\varphi \in \mathcal{C}^{2}(\Omega, \mathbb{R})$ and a point $x_{\mathrm{o}} \in \Omega$ such that $w-\varphi<0$ in $\Omega \backslash\left\{x_{\mathrm{o}}\right\}, w\left(x_{\mathrm{o}}\right)=\varphi\left(x_{\mathrm{o}}\right)$, and $\left(\Delta_{F}\right)_{*}[\varphi]\left(x_{\mathrm{o}}\right)>0$. By lower semicontinuity, we can fix $c>0$ and find a $\delta=\delta(c)>0$ such that $\delta<\operatorname{inj}\left(x_{\mathrm{o}}\right), B\left(x_{\mathrm{o}}, \delta\right) \subset \Omega$ and

$$
\left(\Delta_{F}\right)_{*}[\varphi](x)>2 c \quad \text { for any } x \in B\left(x_{\mathrm{o}}, \delta\right) .
$$

Set $v_{\varphi}:=v+\varphi \in \operatorname{LSC}(\Omega)$ and observe that $v_{\varphi}$ is a viscosity supersolution to $F[u]-2 c=0$ on the ball $B\left(x_{0}, \delta\right)$. Indeed, given $x \in B\left(x_{0}, \delta\right)$ and a function $\psi \in \mathcal{C}^{2}\left(B\left(x_{0}, \delta\right), \mathbb{R}\right)$ such that $v_{\varphi}-\psi \geq 0$ around $x$ and $v_{\varphi}(x)=\psi(x)$, from the very definition of $v_{\varphi}$ we get $v-(\psi-\varphi) \geq 0$ around $x$ and $v(x)=\psi(x)-\varphi(x)$. Hence, being $v$ a viscosity supersolution of $F[u]=0$ on $\Omega$, from the very definition of $\Delta_{F}$ we get

$$
\begin{aligned}
& F[\psi](x) \geq F\left(x, \psi(x)-\varphi(x), \mathrm{d} \psi(x)-\mathrm{d} \varphi(x), D^{2} \psi(x)-D^{2} \varphi(x)\right) \\
& \quad+\left(\Delta_{F}\right)_{*}\left(x, \varphi(x), \mathrm{d} \varphi(x), D^{2} \varphi(x)\right) \geq 2 c .
\end{aligned}
$$

Let us define now

$$
F_{C}: J^{2} M \rightarrow \mathbb{R}, \quad F_{C}(x, s, q, Q):=F(x, s, q, Q)+(C+1) s .
$$

Then, by hypothesis, it follows that:

- $v_{\varphi}$ is a viscosity supersolution of $F_{C}[u]-(C+1) u-2 c=0$ on $\Omega$;

- $u$ is a viscosity subsolution of $F_{C}[u]-(C+1) u=0$ on $\Omega$. 
Since $u \leq v_{\varphi}$ and $u \in \operatorname{USC}(\Omega), v_{\varphi} \in \operatorname{LSC}(\Omega)$, by using the Hahn-Katetov-Dowker Theorem (see [18, Thm 3.4.9]), combined with an approximation argument, it follows that there exists a Lipschitz continuous function $h: \bar{B}\left(x_{\mathrm{o}}, \delta\right) \rightarrow \mathbb{R}$ such that

$$
(C+1) u(x) \leq h(x) \leq(C+1) v_{\varphi}(x)+c \quad \text { for any } x \in \bar{B}\left(x_{0}, \delta\right) .
$$

Therefore:

- $v_{\varphi}$ is a viscosity supersolution of $F_{C}[u]-h(x)-c=0$ on $B\left(x_{0}, \delta\right)$;

- $u$ is a viscosity subsolution of $F_{C}[u]-h(x)=0$ on $B\left(x_{0}, \delta\right)$.

Since by hypothesis $u-v_{\varphi}$ has a strict maximum at $x_{0}$ and $u\left(x_{0}\right)=v_{\varphi}\left(x_{0}\right)$, we can take $k>0$ such that

$$
u-v_{\varphi} \leq-k \text { on } S\left(x_{0}, \delta\right) \text { and }(2 C+1) k \leq c
$$

Then, we take $v_{\varphi, k}:=v_{\varphi}-k$ so that $v_{\varphi, k} \geq u$ on $S\left(x_{\mathrm{o}}, \delta\right)$ and $v_{\varphi, k}\left(x_{\mathrm{o}}\right)=u\left(x_{\mathrm{o}}\right)-k<$ $u\left(x_{\mathrm{o}}\right)$. Note that, since $F$ is Lipschitz with constant $C$, the operator $F_{C}$ verifies

$$
F_{C}(x, r-k, q, Q) \geq F_{C}(x, r, q, Q)-(2 C+1) k \text {. }
$$

Therefore, using then the fact that $v_{\varphi}$ is a viscosity supersolution of $F_{C}[u]-h(x)-$ $c=0$ on $B\left(x_{0}, \delta\right)$ and the inequality $-(2 C+1) k \geq-c$, we conclude that the perturbation $v_{\varphi, k}$ is a viscosity supersolution to $F_{C}[u]-h(x)=0$ on $B\left(x_{0}, \delta\right)$. It is now immediate to observe that the operator $F_{C}$ verifies the hypotheses of the weak Comparison Principle [4, Cor 4.8]. In particular, it satisfies condition (1) in [4, Cor 4.8] (with $\gamma=1$ ), since (u.l.p.) implies that $r \mapsto F_{C}(x, r, q, Q)-r$ is non-decreasing on $\mathbb{R}$. Then, by applying [4, Cor 4.8], we can conclude that $v_{\varphi, k} \geq u$ in $B\left(x_{0}, \delta\right)$, which leads to the contradiction $k \leq 0$.

Finally, we are ready to prove Theorem 5.4.

Proof of Theorem 5.4 By Lemma 5.5, we know that $w$ is a viscosity subsolution of $\left(\Delta_{F}\right)_{*}[u]=0$. Then, we consider $G: J^{2} M \rightarrow \mathbb{R}$ given by $G(x, s, q, Q):=$ $\mathcal{M}_{\lambda, \Lambda}^{+}(x, Q)+C(|s|+|q|)$ and we observe that

$$
-\left(\Delta_{F}\right)_{*}(x,-s,-q,-Q) \leq G(x, s, q, Q) \text { for any }(x, s, q, Q) \in J^{2} M .
$$

Hence, it follows that $-w$ is a supersolution of $G[u]=0$. Since $G$ is continuous, uniformly elliptic and positively 1-homogeneous, the result is a consequence of Theorem 4.7 .

Remark 5.6 The result holds true for equations driven by the normalized $p$-Laplacian on Riemannian manifolds under the assumptions of Theorem 5.4.

Remark 5.7 The assumption on the sectional curvature of $(M, g)$ can be dropped at the expenses of assuming a further uniform continuity assumption in the variable $x$ and $D^{2} u$ (see [4, Cor 4.10]). More precisely, Theorem 5.4 holds true in a general Riemannian manifold, without curvature conditions, if we replace the intrinsic uniform continuity (i.u.c.) by: 
(i.u.c.') for any $\varepsilon>0$ there exists $\delta>0$ such that, for any $x, y \in M$ with dist $(x, y)<\min \{\operatorname{inj}(x), \operatorname{inj}(y)\}$, it holds

$$
\begin{aligned}
& \operatorname{dist}(x, y) \leq \delta, \quad-\delta I \leq P-L_{x y}^{*} Q \leq \delta I \\
& \Longrightarrow\left|F(y, s, q, Q)-F\left(x, s, L_{x y}^{*} q, P\right)\right| \leq \varepsilon
\end{aligned}
$$

for any $(s, q, P) \in J_{y}^{2} M, Q \in \operatorname{Sym}^{2}\left(T_{x}^{*} M\right)$.

Funding Open Access funding provided by Università degli Studi di Padova.

Open Access This article is licensed under a Creative Commons Attribution 4.0 International License, which permits use, sharing, adaptation, distribution and reproduction in any medium or format, as long as you give appropriate credit to the original author(s) and the source, provide a link to the Creative Commons licence, and indicate if changes were made. The images or other third party material in this article are included in the article's Creative Commons licence, unless indicated otherwise in a credit line to the material. If material is not included in the article's Creative Commons licence and your intended use is not permitted by statutory regulation or exceeds the permitted use, you will need to obtain permission directly from the copyright holder. To view a copy of this licence, visit http://creativecommons.org/licenses/by/4.0/.

\section{References}

1. Alías, L.J., Mastrolia, P., Rigoli, M.: Maximum Principles and Geometric Applications. Springer Monographs in Mathematics. Springer, Cham (2016)

2. Amendola, M.E., Rossi, L., Vitolo, A.: Harnack inequalities and ABP estimates for nonlinear secondorder elliptic equations in unbounded domains. Abstr. Appl. Anal., Art. ID 178534, 19 (2008)

3. Antonini, P., Mugnai, D., Pucci, P.: Quasilinear elliptic inequalities on complete Riemannian manifolds. J. Math. Pures Appl. 87(6), 582-600 (2007)

4. Azagra, D., Ferrera, J., Sanz, B.: Viscosity solutions to second order partial differential equations on Riemannian manifolds. J. Differ. Equ. 245(2), 307-336 (2008)

5. Bardi, M., Capuzzo-Dolcetta, I.: Optimal Control and Viscosity Solutions of Hamilton-Jacobi-Bellman Equations. Systems \& Control: Foundations \& Applications. Birkhäuser, Boston (1997)

6. Bardi, M., Cesaroni, A.: Liouville properties and critical value of fully nonlinear elliptic operators. J. Differ. Equ. 261(7), 3775-3799 (2016)

7. Bardi, M., Da Lio, F.: On the strong maximum principle for fully nonlinear degenerate elliptic equations. Arch. Math. (Basel) 73(4), 276-285 (1999)

8. Bardi, M., Da Lio, F.: Propagation of maxima and strong maximum principle for viscosity solutions of degenerate elliptic equations. I. Convex operators. Nonlinear Anal. 44, 991-1006 (2001)

9. Bardi, M., Da Lio, F.: Propagation of maxima and strong maximum principle for viscosity solutions of degenerate elliptic equations. II. Concave operators. Indiana Univ. Math. J. 52(3), 607-627 (2003)

10. Bardi, M., Goffi, A.: New strong maximum and comparison principles for fully nonlinear degenerate elliptic PDEs. Calc. Var. Partial Differ. Equ. 58(6), 20 (2019)

11. Bardi, M., Mannucci, P.: On the Dirichlet problem for non-totally degenerate fully nonlinear elliptic equations. Commun. Pure Appl. Anal. 5(4), 709-731 (2006)

12. Barles, G., Busca, J.: Existence and comparison results for fully nonlinear degenerate elliptic equations without zeroth-order term. Commun. Partial Differ. Equ. 26(11-12), 2323-2337 (2001)

13. Besse, A.L.: Einstein manifolds. Classics in Mathematics. Springer, Berlin, 2008. Reprint of the 1987 edition

14. Bianchini, B., Mari, L., Pucci, P., Rigoli, M.: On the interplay among maximum principles, compact support principles and Keller-Osserman conditions on Manifolds. arXiv:1801.02102 (2018)

15. Birindelli, I., Demengel, F.: Comparison principle and Liouville type results for singular fully nonlinear operators. Ann. Fac. Sci. Toulouse Math. 13(2), 261-287 (2004)

16. Birindelli, I., Galise, G., Ishii, H.: A family of degenerate elliptic operators: maximum principle and its consequences. Ann. Inst. H. Poincaré Anal. Non Linéaire 32(2), 417-441 (2020) 
17. Birindelli, I., Galise, G., Ishii, H.: Positivity sets of supersolutions of degenerate elliptic equations and the strong maximum principle. Trans. Am. Math. Soc. (2020). https://doi.org/10.1090/tran/8226

18. Borwein, J.M., Vanderwerff, J.D.: Convex Functions: Constructions, characterizations and counterexamples. Encyclopedia of Mathematics and Its Applications, vol. 109. Cambridge University Press, Cambridge (2010)

19. Burago, D., Burago, Y., Ivanov, S.: A Course in Metric Geometry. Graduate Studies in Mathematics, vol. 33. American Mathematical Society, Providence (2001)

20. Cabré, X.: Nondivergent elliptic equations on manifolds with nonnegative curvature. Commun. Pure Appl. Math. 50(7), 623-665 (1997)

21. Caffarelli, L.A., Cabré, X.: Fully Nonlinear Elliptic Equations. American Mathematical Society Colloquium Publications, vol. 43. American Mathematical Society, Providence (1995)

22. Caffarelli, L., Li, Y., Nirenberg, L.: Some remarks on singular solutions of nonlinear elliptic equations III: viscosity solutions including parabolic operators. Commun. Pure Appl. Math. 66(1), 109-143 (2013)

23. Calabi, E.: An extension of E. Hopf's maximum principle with an application to Riemannian geometry. Duke Math. J. 25, 45-56 (1958)

24. Chow, B., Chu, S.-C., Glickenstein, D., Guenther, C., Isenberg, J., Ivey, T., Knopf, D., Lu, P., Luo, F., Ni, L.: The Ricci flow: techniques and applications. Part II, vol. 44 Mathematical Surveys and Monographs. American Mathematical Society, Providence (2008)

25. Cirant, M., Harvey, F.R., Lawson, Jr. H.B., Payne, K.: Comparison principles by monotonicity and duality for constant coefficient nonlinear potential theory and PDEs. arXiv:2009.01611 (2020)

26. Crandall, M.G., Ishii, H., Lions, P.-L.: User's guide to viscosity solutions of second order partial differential equations. Bull. Am. Math. Soc. 27(1), 1-67 (1992)

27. Felmer, P., Quaas, A., Sirakov, B.: Solvability of nonlinear elliptic equations with gradient terms. J. Differ. Equ. 254(11), 4327-4346 (2013)

28. Ferrari, F., Vitolo, A.: Regularity properties for a class of non-uniformly elliptic Isaacs operators. Adv. Nonlinear Stud. 20(1), 213-241 (2020)

29. Giga, Y.: Surface Evolution Equations. Monographs in Mathematics. A Level Set Approach, vol. 99. Birkhäuser, Basel (2006)

30. Giga, Y., Ohnuma, M.: On strong comparison principle for semicontinuous viscosity solutions of some nonlinear elliptic equations. Int. J. Pure Appl. Math. 22(2), 165-184 (2005)

31. Gilbarg, D., Trudinger, N.S.: Elliptic partial differential equations of second order. Grundlehren der Mathematischen Wissenschaften [Fundamental Principles of Mathematical Sciences], vol. 224, 2nd edn. Springer, Berlin (1983)

32. Grigor'yan, A.: Analytic and geometric background of recurrence and non-explosion of the Brownian motion on Riemannian manifolds. Bull. Am. Math. Soc. 36(2), 135-249 (1999)

33. Grigor'yan, A.: Heat Kernel and Analysis on Manifolds. AMS/IP Studies in Advanced Mathematics, vol. 47. American Mathematical Society, Providence (2009)

34. Harvey, F.R., Lawson Jr., H.B.: Dirichlet duality and the nonlinear Dirichlet problem on Riemannian manifolds. J. Differ. Geom. 88(3), 395-482 (2011)

35. Harvey, F.R., Lawson Jr., H.B.: Characterizing the strong maximum principle for constant coefficient subequations. Rend. Mat. Appl. 37(1-2), 63-104 (2016)

36. Ishii, H., Yoshimura, Y.: Demi-eigenvalues for uniformly elliptic Isaacs operators. unpublished manuscript

37. Jensen, R.: Uniqueness criteria for viscosity solutions of fully nonlinear elliptic partial differential equations. Indiana Univ. Math. J. 38(3), 629-667 (1989)

38. Juutinen, P., Lindqvist, P., Manfredi, J.J.: On the equivalence of viscosity solutions and weak solutions for a quasi-linear equation. SIAM J. Math. Anal. 33(3), 699-717 (2001)

39. Kawohl, B., Kutev, N.: Strong maximum principle for semicontinuous viscosity solutions of nonlinear partial differential equations. Arch. Math. (Basel) 70(6), 470-478 (1998)

40. Kawohl, B., Kutev, N.: Comparison principle for viscosity solutions of fully nonlinear, degenerate elliptic equations. Commun. Partial Differ. Equ. 32(7-9), 1209-1224 (2007)

41. Kim, S.: Harnack inequality for degenerate and singular operators of $p$-Laplacian type on Riemannian manifolds. Math. Ann. 366(3-4), 1721-1785 (2016)

42. Li, Y., Wang, B.: Strong comparison principles for some nonlinear degenerate elliptic equations. Acta Math. Sci. Ser. B (Engl. Ed.) 38(5), 1583-1590 (2018) 
43. Mari, L., Pessoa, L.F.: Duality between Ahlfors-Liouville and Khas'minskii properties for non-linear equations. Commun. Anal. Geom. 28(2), 395-497 (2020)

44. Mohammed, A., Vitolo, A.: On the strong maximum principle. Complex Var. Elliptic Equ. 65(8), 1299-1314 (2020)

45. Ohnuma, M., Sakaguchi, S.: A simple proof of a strong comparison principle for semicontinuous viscosity solutions of the prescribed mean curvature equation. Nonlinear Anal. 181, 180-188 (2019)

46. Patrizi, S.: Principal eigenvalues for Isaacs operators with Neumann boundary conditions. NoDEA 16(1), 79-107 (2009)

47. Pediconi, F.: A local version of the Myers-Steenrod theorem. Bull. Lond. Math. Soc. 52(5), 871-884 (2020)

48. Pessoa, L.F., Pigola, S., Setti, A.G.: Dirichlet parabolicity and $L^{1}$-Liouville property under localized geometric conditions. J. Funct. Anal. 273(2), 652-693 (2017)

49. Pigola, S., Rigoli, M., Setti, A.G.: Maximum principles on Riemannian manifolds and applications. Mem. Am. Math. Soc., 174(822):x+99 (2005)

50. Protter, M.H., Weinberger, H.F.: Maximum Principles in Differential Equations. Springer, New York, 1984. Corrected reprint of the 1967 original

51. Pucci, C.: Operatori ellittici estremanti. Ann. Mater. Pura Appl. 4(72), 141-170 (1966)

52. Pucci, P., Serrin, J.: The strong maximum principle revisited. J. Differ. Equ. 196(1), 1-66 (2004)

53. Pucci, P., Serrin, J.: The Maximum Principle. Progress in Nonlinear Differential Equations and Their Applications, vol. 73. Birkhäuser, Basel (2007)

54. Punzo, F.: Liouville theorems for fully nonlinear elliptic equations on spherically symmetric Riemannian manifolds. NoDEA 20(3), 1295-1315 (2013)

55. Sakai, T.: On continuity of injectivity radius function. Math. J. Okayama Univ. 25(1), 91-97 (1983)

56. Trudinger, N.S.: Comparison principles and pointwise estimates for viscosity solutions of nonlinear elliptic equations. Rev. Mat. Iberoamericana 4(3-4), 453-468 (1988)

57. Vázquez, J.L.: A strong maximum principle for some quasilinear elliptic equations. Appl. Math. Optim. 12(3), 191-202 (1984)

58. Vitolo, A.: Singular elliptic equations with directional diffusion. Math. Eng., 3(3):Paper No. 027,16 (2021)

59. $\mathrm{Xu}, \mathrm{S}$.: Local estimate on convexity radius and decay of injectivity radius in a Riemannian manifold. Commun. Contemp. Math. 20(6), 1750060 (2018)

Publisher's Note Springer Nature remains neutral with regard to jurisdictional claims in published maps and institutional affiliations. 